ARTICLE

\title{
Conserved long-range base pairings are associated with pre-mRNA processing of human genes
}

Svetlana Kalmykova', Marina Kalinina (1) ${ }^{1}$, Stepan Denisov ${ }^{1}$, Alexey Mironov', Dmitry Skvortsov², Roderic Guigó (iD ${ }^{3} \&$ Dmitri Pervouchine ${ }^{1 凶}$

The ability of nucleic acids to form double-stranded structures is essential for all living systems on Earth. Current knowledge on functional RNA structures is focused on locallyoccurring base pairs. However, crosslinking and proximity ligation experiments demonstrated that long-range RNA structures are highly abundant. Here, we present the most complete todate catalog of conserved complementary regions (PCCRs) in human protein-coding genes. PCCRs tend to occur within introns, suppress intervening exons, and obstruct cryptic and inactive splice sites. Double-stranded structure of PCCRs is supported by decreased icSHAPE nucleotide accessibility, high abundance of RNA editing sites, and frequent occurrence of forked eCLIP peaks. Introns with PCCRs show a distinct splicing pattern in response to RNAPII slowdown suggesting that splicing is widely affected by co-transcriptional RNA folding. The enrichment of 3 '-ends within PCCRs raises the intriguing hypothesis that coupling between RNA folding and splicing could mediate co-transcriptional suppression of premature pre-mRNA cleavage and polyadenylation.

\footnotetext{
${ }^{1}$ Skolkovo Institute of Science and Technology, Moscow, Russia. ${ }^{2}$ Faculty of Chemistry, Moscow State University, Moscow, Russia. ${ }^{3}$ Center for Genomic Regulation and UPF, Barcelona, Spain. ${ }^{凶}$ email: d.pervouchine@skoltech.ru
} 
A double-stranded structure is a key feature of nucleic acids that enables replicating the genomic information and underlies fundamental cellular processes ${ }^{1,2}$. Many RNAs adopt functional secondary structures, and messenger RNAs (mRNAs) are no exception, although their main role is to encode proteins $^{3-6}$. In eukaryotes, RNA structure affects gene expression through modulating all steps of pre-mRNA processing including splicing $^{7}$, cleavage and polyadenylation ${ }^{8}$, and RNA editing ${ }^{9}$. The loss of functional RNA structure has been increasingly reported as implicated in human disease $\mathrm{s}^{10-13}$.

To date, a few dozens of functional RNA structures have been characterized in the human genome (Table 1 and Fig. S1). Many of them are formed between evolutionarily conserved regions that are located in introns of protein-coding genes and consist of base pairings spanning thousands of nucleotides. Computational identification of such distant base pairings by de novo RNA folding is not feasible due to a number of technical limitations ${ }^{14}$. However, recent progress in high-throughput sequencing techniques enabled novel experimental strategies to determine RNA structure in vivo ${ }^{15-19}$. In particular, photo-inducible RNA cross-linking and proximity ligation assays revealed that long-range base pairings are highly abundant in the human transcriptome ${ }^{20-24}$. Currently, the applicability of these assays for large-scale profiling of RNA structure is still limited, and computational identification of long-range RNA structure remains a great challenge in RNA biology.

Comparative genomics provides a powerful alternative to de novo RNA folding by detecting signatures of evolutionary conservation ${ }^{25,26}$. Previous reports presented complex methodologies that implement simultaneous alignment and folding to detect RNA elements with divergent sequences that are nevertheless conserved at the secondary structure level ${ }^{27-30}$. However, a substantial fraction $(3.4 \%)$ of intronic nucleotides in the human genome are highly conserved at the sequence level, which raises a compelling question of whether their function may be related to RNA structure. This motivated us to revisit this problem with the "first-align-then-fold" approach ${ }^{14}$, one which finds pairs of conserved complementary regions (PCCRs) in pre-aligned evolutionarily conserved regions. In this study, we developed a method named PrePH (PREdiction of PanHandles) to efficiently find long, nearly perfect complementary matches in a pair of input sequences, and applied it to all pairwise combinations of CIRs located at a certain distance limit from each other. Subsequently, we analyzed MSAs that had a sufficient amount of variation to detect compensatory substitutions within PCCRs.

Multiple lines of evidence indicate that a large proportion of PCCRs indeed have a double-stranded structure, for example, significant decrease of icSHAPE nucleotide accessibility, high abundance of adenosine-to-inosine (A-to-I) RNA-editing sites, significant overlap with long-range RNA contacts identified by proximity ligation assays, and frequent co-occurrence of the socalled forked enhanced cross-linking and immunoprecipitation
(eCLIP) peaks (see below). They also have other characteristic features such as occurrence within introns proximally to splice sites, avoidance of branch points, and obstructive effect on cryptic and inactive splice sites. At the same time, the method has a substantial false-positive rate, which, as we show, cannot be reduced without losing sensitivity to bona fide validated RNA structures (Table 1). The catalog of PCCRs is provided as a reference dataset that is conveniently visualized through a UCSC Genome Browser track hub ${ }^{31}$. We additionally provide a transcriptome-wide characterization of RNA bridges ${ }^{32}$ and exon loop-outs ${ }^{33}$, two particular mechanisms of alternative splicing regulation by long-range RNA structures.

\section{Results}

Conserved complementary regions. To identify conserved complementary regions (CCRs), we considered nucleotide sequences of human protein-coding genes excluding all constitutive and alternative exons, repeats, noncoding genes residing in introns, and other regions with selective constraints that may not be related to base pairings (Fig. 1A). The remaining intronic regions were extended by $10 \mathrm{nts}$ (nucleotides) to within flanking exons to allow for base pairings that overlap splice sites. The resulting set of 236,332 intronic regions was intersected with the phastConsElements track of the UCSC Genome Browser ${ }^{34}$, which defines genomic intervals that are highly conserved between 100 vertebrates (major species Pan troglodytes, Mus musculus, Sus scrofa, Gallus gallus, Xenopus tropicalis, Dani rerio; the shortest and the longest phylogenetic distances 0.01 and 2.40, respectively). This resulted in a set of 1,931,116 short fragments with the median length $17 \mathrm{nts}$, which will be referred to as conserved intronic regions (CIRs).

PCCRs were identified in all pairwise combinations of CIR that are located not more than $L$ nucleotides apart from each other and belong to the same gene using PrePH, a $k$-mer-based method (Fig. 1B) that efficiently predicts long, nearly perfect stretches of complementary nucleotides in a pair of input sequences (see "Methods"). A search for at least 10-nt-long sequences with the hybridization free energy $\Delta G \leq-15 \mathrm{kcal} / \mathrm{mol}$, minimum helix length $k \geq 5$, and distance limit $L \leq 10,000$ yielded 916,360 PCCRs, on average 75 PCCR per gene, with $95 \%$ of genes having not more than 295 PCCRs (Supplementary Data Files 1 and 2). The median free energy of hybridization $(\Delta G)$ and the median length of CCRs were $-17.2 \mathrm{kcal} / \mathrm{mol}$ and $13 \mathrm{nts}$, respectively, with the frequency distribution decaying towards longer and more stable structures (Fig. 1C and Fig. S1A). As expected, longer structures had larger absolute values of $\Delta G$; however, the energy and the length of a PCCR are not directly proportional and the relationship between them depends on the GC content (Fig. S1B, S1C). In what follows, PCCRs are classified into four energy groups, group I from -15 to $-20 \mathrm{kcal} / \mathrm{mol}$, group II from -20 to $-25 \mathrm{kcal} / \mathrm{mol}$, group III from -25 to $-30 \mathrm{kcal} / \mathrm{mol}$, and group IV

Table 1 Experimentally validated (bona fide) RNA structures in human genes that satisfied PrePH search criteria.

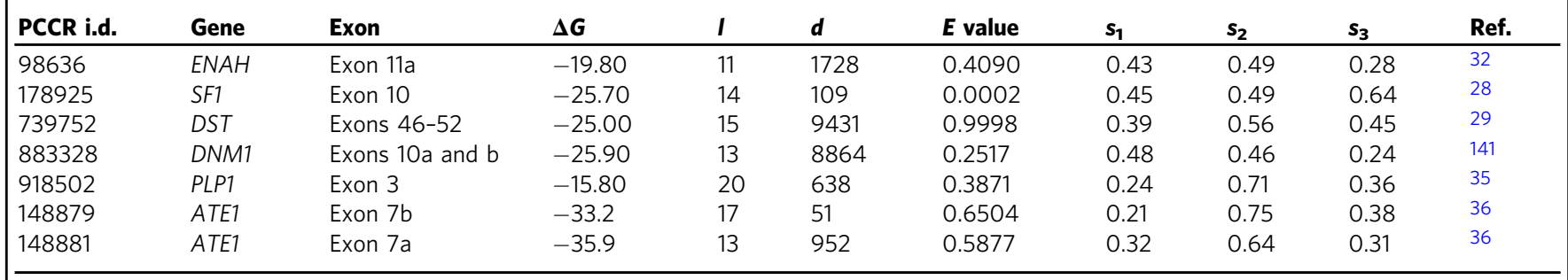

$\Delta G$ predicted free energy $(\mathrm{kcal} / \mathrm{mol})$, I average length of the two CCRs (nts), $d$ spread (distance between complementary regions, nts), E value $E$ value from R-scape after correction for multiple testing, $s$, $\mathrm{s}_{2}, \mathrm{~s}_{3}$ nucleotide conservation metrics (see "Compensatory substitutions" section). 
A

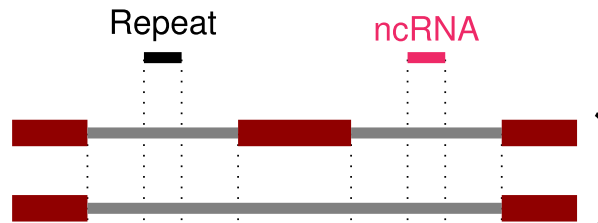

protein-coding

transcripts

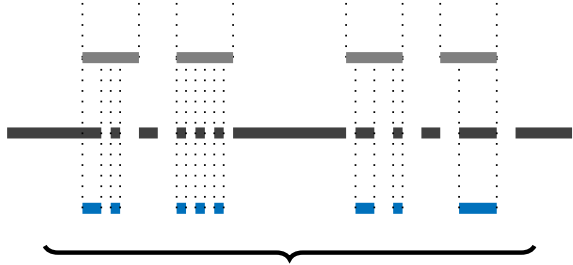

all-to-all pairs within $10 \mathrm{~Kb}$

intronic

phastCons elements

CIR
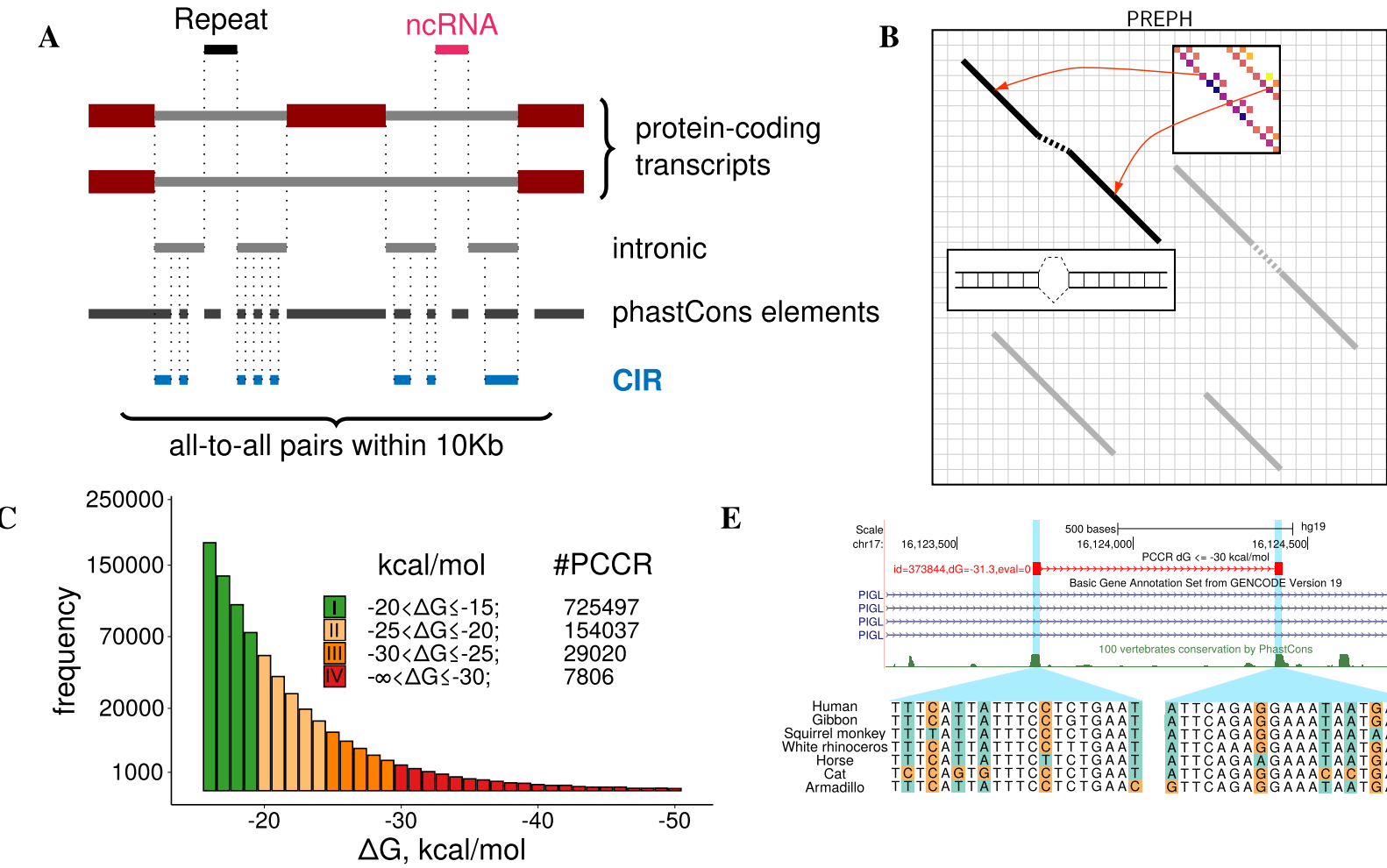

$\mathbf{E}$

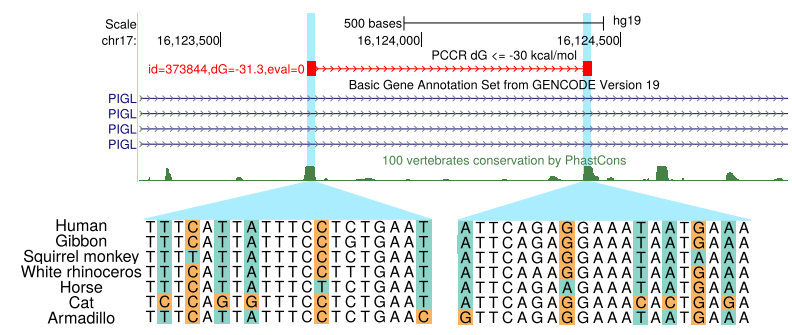

D

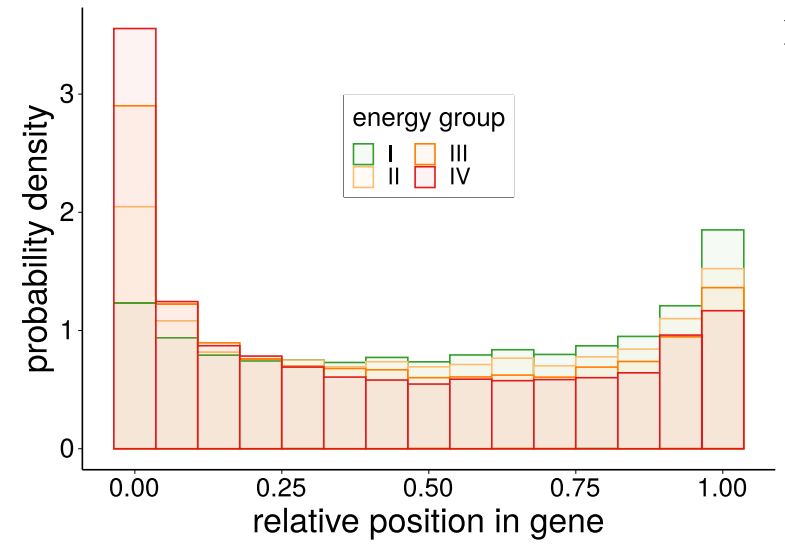

F

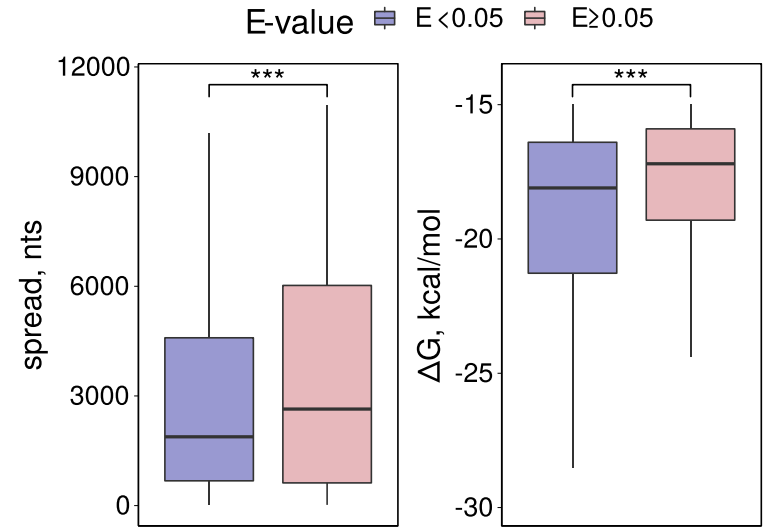

Fig. 1 Pairs of conserved complementary regions (PCCRs). A PCCRs are identified in conserved intronic regions (CIRs) that are $<10,000$ nts apart from each other. B PrePH computes the dynamic programming matrix based on the precomputed helix energies for all $k$-mers (inset) and energies of short internal loops and bulges (see Supplementary Methods for details). C The distribution of PCCR energies consists of four energy groups: group I $(-20<\Delta G \leq-15 \mathrm{kcal} / \mathrm{mol})$, group II $(-25<\Delta G \leq-20 \mathrm{kcal} / \mathrm{mol})$, group III $(-30<\Delta G \leq-25 \mathrm{kcal} / \mathrm{mol})$, and group IV $(\Delta G \leq-30 \mathrm{kcal} / \mathrm{mol})$. D The distribution of $p$, relative position of a PCCR in the gene. E Multiple independent compensatory substitutions support long-range RNA structure in the phosphatidylinositol glycan anchor biosynthesis class L (PIGL) gene. F PCCRs with significant nucleotide covariations $(E$ value $<0.05, n=3204)$ are on average less spread and more stable than PCCRs with insignificant nucleotide covariations ( $E$ value $\geq 0.05, n=905942)$; two-sided Mann-Whitney test; ${ }^{\star \star \star}$ denotes a statistically discernible difference at the $0.1 \%$ significance level.

below $-30 \mathrm{kcal} / \mathrm{mol}$, which are represented throughout the paper by a uniform color scheme shown in Fig. 1C.

To assess the sensitivity of the method with respect to known RNA structures, we collected evidence of functional base pairings in human genes from the literature. All bona fide structures that satisfied the search criteria were successfully found (Table 1 and Fig. S2), while others either did not pass the free energy cutoff, were shorter than $10 \mathrm{nts}$, located outside of CIR, or did not belong to protein-coding genes (Table S1). Notably, the long-distance intronic interaction that regulates PLP1/DM20 splicing $^{35}$ and the RNA bridge in $E N A H^{32}$ were both assigned to group I ( $\triangle G=$ -15.8 and $-19.4 \mathrm{kcal} / \mathrm{mol}$, respectively), indicating that less stable structures are not less functional or less interesting than the others. For the purpose of presentation here, we chose the distance limit $L=10,000$, which by the order of magnitude corresponds to long-range RNA structures listed in Table 1. However, our recent study of the human ATE1 gene demonstrated that functional RNA structures may spread over much longer distances ${ }^{36}$. We, therefore, additionally explored how the number of PCCRs changes with increasing the distance limit and found that it grows approximately fourfold with increasing $L$ up to 100,000 nts (Fig. S3A).

The frequency distribution of the distances between two CCRs in a pair, here referred to as spread, peaks at short distances, decaying towards a non-zero baseline (Fig. S3B). This baseline originates from the distribution of pairwise distances between CIR that were passed to PrePH as an input, which decays towards the same baseline. We next asked whether PCCRs were 
distributed uniformly along the gene, or they tend to accumulate in certain gene parts. To quantify the position of a PCCR within a gene, we introduced $p$, a measure of relative position, which changes from $0 \%$ for the regions located in the very $5^{\prime}$ end of the gene to $100 \%$ for the regions located in the very $3^{\prime}$ end. The metric $p$ can be computed for a PCCR as a whole to represent its relative position, or for each of its CCR separately. The location of PCCR as a whole was not uniform, with two pronounced modes at the $5^{\prime}$ and $3^{\prime}$ end (Fig. 1D). This enrichment was also prominent in the distribution of single CCRs (Fig. S3C), which could be due to stronger evolutionary constraints on the nucleotide sequences at gene ends. Indeed, we found that CCRs exhibit a higher degree of evolutionary conservation than their adjacent regions within CIR (the difference of the average phastCons scores, MW (Mann-Whitney sum of ranks test) test, $p$ value $\left.<2.2 \times 10^{-16}\right)$ and thus tend to occur in more constrained regions. Gene ontology $(\mathrm{GO})$ terms associated with genes that have PCCRs were enriched with terms related to morphogenesis and development of the central nervous system as compared to genes of the same length, but without PCCRs (Fig. S4).

Compensatory substitutions. Compensatory mutations, that is, pairs of nucleotide substitutions that individually disrupt base pairings, but restore them when introduced in combination, play a central role in the evolution of RNA structure $37-39$. To analyze compensatory mutations in PCCRs, we applied the R-scape program ${ }^{40}$, which scores independent occurrence of complementary substitutions on different branches of the phylogenetic tree, to pairs of multiple sequence alignments (MSAs) that were cut out from the MSA of 100 vertebrates $^{34}$ by PCCRs (see "Methods"). The deviation from the null hypothesis that pairwise covariations in a PCCR are not due to conservation of RNA structure was estimated as a product of $E$ values reported by Rscape for all base pairs within the PCCR. These products were adjusted using Benjamini-Hochberg correction.

Out of 916,360 PCCR, for which this computation was possible, only 909,146 had a sufficient number of substitutions to estimate the $E$ value, and only 3204 of them had $E$ value below $5 \%$ (Fig. S5). The PCCRs with $E$ value $<0.05$ were on average more stable and less spread than PCCRs with $E$ value $\geq 0.05$ (Fig. 1F). In some cases, structural alignments were strongly supported by covariations, that is, a highly stable $\operatorname{PCCR}(\Delta G=$ $-31 \mathrm{kcal} / \mathrm{mol}$ ) spanning $700 \mathrm{nts}$ in the first intron of PIGL gene (Fig. 1E) and PCCRs in the QRICH2 and MRPL42 genes (Fig. S6). However, $E$ values of bona fide RNA structures from Table 1 ranged from 0.0002 for the PCCR responsible for splicing of the intron between exons 9 and 10 in SF1 to 0.9998 for the PCCR associated with exons 46-52 skipping in DST, suggesting that the amount of variation in the nucleotide sequences of PCCRs is generally not sufficient to estimate their statistical significance through compensatory substitutions.

A remarkable feature of standalone RNA regulatory elements is their high level of conservation, as opposed to that of their flanking sequences ${ }^{14}$. We introduced three additional metrics to capture the nucleotide conservation rate within CCR as compared to the background: $s_{1}$ is the difference between the average phastCons scores within CCR and within 300 nts flanking regions (the larger, the more significant); $s_{2}$ is the average phastCons score within CCR and $300 \mathrm{nts}$ around it (the smaller, the more significant); $s_{3}$ is the length of a CCR relative to the length of its parent CIR (the larger, the more significant). However, neither of the three metrics reached extreme values for the base pairings listed in Table 1 (Fig. S7), which indicates that functional PCCRs are not necessarily located in isolated conserved regions and may well occur in a relatively conserved background.
Support by high-throughput structural assays. PCCRs represent regions in the pre-mRNA that have an increased capacity to base pair. The propensity of individual nucleotides to base pair was assessed at the transcriptome-wide level by measuring the nucleotide flexibility score with icSHAPE method ${ }^{41}$. We compared the average icSHAPE reactivity within CCR with that in a control set of intervals located nearby (Fig. 2A). Indeed, the average icSHAPE reactivity of nucleotides within CCR was significantly lower as compared to the control (Wilcoxon's test, $P<$ $10^{-60}$ ), and the difference increased by the absolute value with the increase in the structure free energy $\left(\left|\hat{\beta}_{1}\right|=0.03 \pm 0.01\right)$. However, the icSHAPE reactivity scores were available only for 4551 PCCRs representing $0.5 \%$ of the full set. We, therefore, sought for PCCR support in other experimental datasets.

Chemical RNA structure probing can reveal which bases are single- or double-stranded, but it cannot determine which nucleotides form base pairs ${ }^{15-19}$. We, therefore, validated PCCRs against the long-range RNA-RNA interactions that were assessed experimentally by psoralen analysis of RNA interactions and structures (PARIS) ${ }^{22}(n=15,036)$, ligation of interacting RNA followed by high-throughput sequencing (LIGR-seq) $)^{23} \quad(n=$ 551,926 ), and RNA in situ conformation sequencing (RICseq $)^{24}(n=501,144)$. Towards this end, we considered the interacting pairs in the experimental data that were located intramolecularly within CIR not $>10,000 \mathrm{nts}$ apart from each other, and restricted the set of PCCRs to the underlying CIRs that overlap the experimental dataset (see "Methods"). The precision $(P)$ and recall $(R)$ metrics were defined as the proportion of PCCRs supported by the experimental method and the proportion of experimental interactions supported by PCCRs, respectively. In addition, we computed $\pi$, the conditional probability of predicting the interacting CCR partner correctly given that another CCRs in a pair has been predicted correctly (Table 2 ). The best agreement was with respect to the RIC-seq dataset, with precision increasing up to $92 \%$ at the expense of decreasing recall when structure stability increased. The $\pi$ metric confirmed that PCCRs tend to correctly identify the interacting partner given that one of the CCRs has been predicted correctly. In addition, we found that free energies of PCCRs supported by RIC-seq and PARIS were significantly lower than those of PCCRs without experimental support (MW test, $\Delta \Delta G \simeq 1.2 \mathrm{kcal} / \mathrm{mol}$, $p$ value $\left.<10^{-19}\right)$. However, the breadth of these findings is limited by small sizes of the true-positive sets ( $n=1903$ for RIC-seq, $n=$ 777 for LIGR-seq, and $n=969$ for PARIS), because structural assays sparsely cover the transcriptome at cell line-specific conditions and focus on intermolecular interactions.

Finally, we explored how the set of predicted PCCRs relates to a similar list that was reported previously by IRBIS ${ }^{29}$. Unlike PrePH, IRBIS follows the "first-fold-then-align" strategy to simultaneously detect conserved complementarity and sequence homology, but at stricter conditions. Overall, the predictions of the two programs had a large intersection relative to IRBIS predictions indicating that the current method generally outputs a superset of IRBIS predictions both in terms of the number of nucleotides and the number of base pairs (Fig. 2B). The free energies of PCCRs supported by IRBIS were significantly lower than those of other PCCRs (MW test, $\Delta \Delta G \simeq 2.7 \mathrm{kcal} / \mathrm{mol}$, $p$ value $<10^{-30}$ ) reflecting the fact that, unlike IRBIS, PrePH allows for short internal loops and bulges. Nevertheless, a small fraction of IRBIS predictions is missing from the list of PCCRs presented here, which relies on the conserved regions from phastConsElements track of the UCSC Genome Browser ${ }^{34}$. Without this limitation, however, the current approach would be impractical from the computational standpoint, and it was our intention to limit the search to conserved regions at the expense 

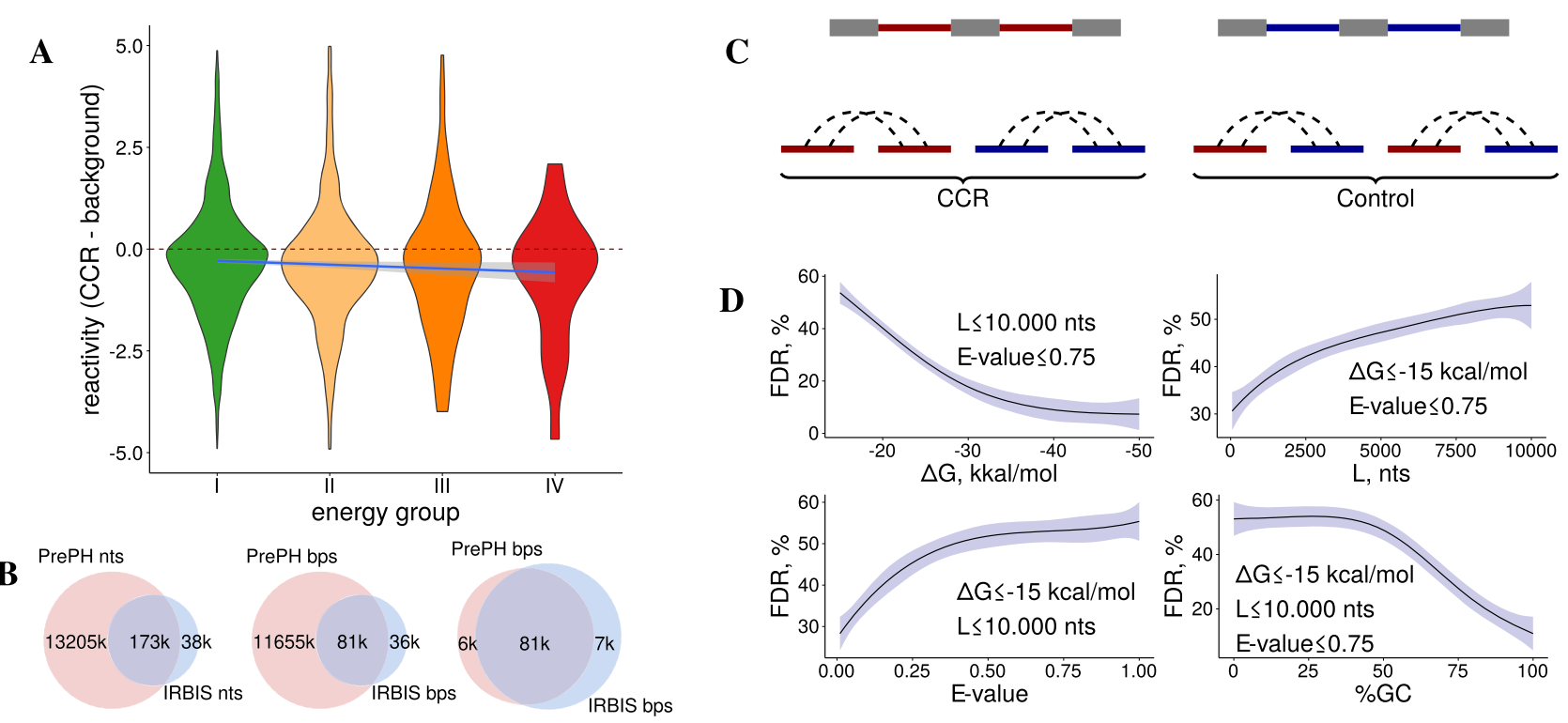

Fig. 2 Validation and false discovery rate (FDR). A The difference between icSHAPE reactivity of nucleotides within CCR and the average reactivity of nearby nucleotides in energy groups I-IV (color code as in Fig. 1C). The linear model $\Delta$ reactivity $=\beta_{0}+\beta_{1} \Delta G$ group is represented by the slanted line; $\hat{\beta}_{1}=-0.03 \pm 0.01$. B Venn diagram for the number of common nucleotides (left), number of common base pairs (middle), and the number of common base pairs among common nucleotides (right) for the predictions of PrePH and IRBIS. C Estimation of the false-positive rate (FDR) by re-wiring, that is, creating a control set that consists of chimeric non-cognate sequences sampled from different genes. D FDR as a function of energy cutoff $\Delta G$ (top left), maximum distance between CIR (top right), $E$ value (bottom left), and GC content (bottom right). Solid lines represent the fitted average over $n=16$ randomizations; shaded areas represent $95 \%$ confidence intervals obtained by the locally estimated scatterplot smoothing (LOESS) regression.

Table 2 Precision and recall at different free energy cutoffs $(\Delta G)$.

\begin{tabular}{|c|c|c|c|c|c|c|c|c|c|c|c|c|}
\hline \multirow[t]{2}{*}{$\Delta \boldsymbol{G}$} & \multicolumn{4}{|c|}{ RIC-seq, $n=1804$} & \multicolumn{4}{|c|}{ LIGR-seq, $n=586$} & \multicolumn{4}{|c|}{ PARIS, $n=907$} \\
\hline & PrePH & $P(\%)$ & $R(\%)$ & $\pi(\%)$ & PrePH & $P(\%)$ & $\boldsymbol{R}(\%)$ & $\pi(\%)$ & PrePH & P (\%) & R (\%) & $\pi(\%)$ \\
\hline-15 & 1611 & 42 & 53 & 43 & 362 & 54 & 49 & 56 & 5901 & 50 & 60 & 51 \\
\hline-25 & 84 & 86 & 9 & 86 & 21 & 48 & 3 & 48 & 545 & 65 & 14 & 65 \\
\hline-30 & 23 & 91 & 3 & 91 & 5 & 40 & 1 & 40 & 160 & 72 & 6 & 73 \\
\hline
\end{tabular}

The precision $(P)$ and recall $(R)$ are the proportion of PCCRs supported by the experimental method and the proportion of experimental interactions supported by PCCRs, respectively. $\pi$ is the conditional probability of predicting the interacting CCR partner correctly given that another CCR in a pair has been predicted correctly. The column "PrePH" shows the number of PCCRs that satisfy the criteria for comparison. The number of structures in each experimental method is denoted by $n$.

of losing some structures that are misaligned by structureagnostic phylogenetic analysis.

False discovery rate. One way to estimate the rate of falsepositive predictions is to apply the same pipeline that was used to identify PCCRs to a control set of sequences that should not base pair. In the previous work, it was described as the so-called "rewiring" approach ${ }^{29}$. Here, we used the same strategy by running the pipeline on chimeric sets of sequences that were sampled randomly from different genes while controlling for nucleotide composition and length, which confound this comparison (Fig. 2C). The false discovery rate (FDR), defined as the number of predictions in the control set as a fraction of the total number of predictions, depends on PCCR energy, spread, GC content, and $E$ value, ranging from $10 \%$ in the most strict to over $50 \%$ in the most relaxed conditions (Fig. 2D). As expected, FDR drops with increasing PCCR energy and GC content and with decreasing PCCR spread and $E$ value.

Many PCCRs listed in Table 1 belong to group I indicating that folding energy alone cannot be used as a threshold to control FDR. To check whether FDR can be improved by simultaneous application of several filters, we applied the most stringent thresholds that preserve PCCRs listed in Table $1(\Delta G \leq-15.8, E$ value $<0.998, \mathrm{GC} \geq 33.3 \%, s_{1} \geq 0.24, s_{2} \leq 0.71$, and $\left.s_{3} \geq 0.24\right)$. The resulting FDR figure of $47 \%$ indicates that FDR cannot be improved without loss of sensitivity with respect to bona fide structures. On the one hand, it implies that more than half of the group I predictions could be false positives. On the other hand, this is a pessimistic estimate since the re-wiring method is known to greatly overestimate the $\mathrm{FDR}^{29}$. We, therefore, interpret $47 \%$ as an excessively conservative FDR estimate and proceed to the statistical characterization of the full PCCR set with the mindset that at least half of the predictions are true positives.

Splicing. Previous reports indicate that long-range base pairings are positioned nonrandomly with respect to splicing signals ${ }^{27-29}$. To elaborate on this, we used the classification shown in Fig. 3A. If a PCCR overlaps an intron, it can be located either entirely within the intron (inside) or the intron can be located entirely within PCCR (outside), or the two intervals intersect (crossing). The tendency of RNA structure to prefer one of these categories is measured by the enrichment metric, defined as the number of PCCRs in the given category relative to the number of PCCR-like intervals in it, computed for a certain control set (each PCCR may 
A

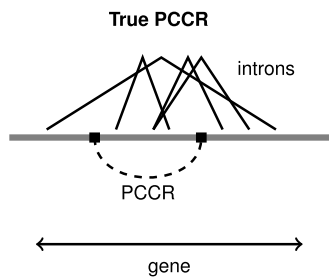

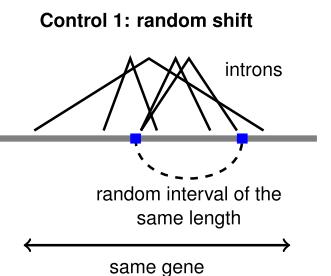

same gene

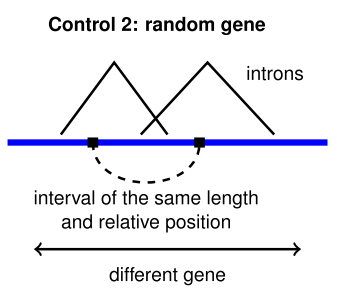

B

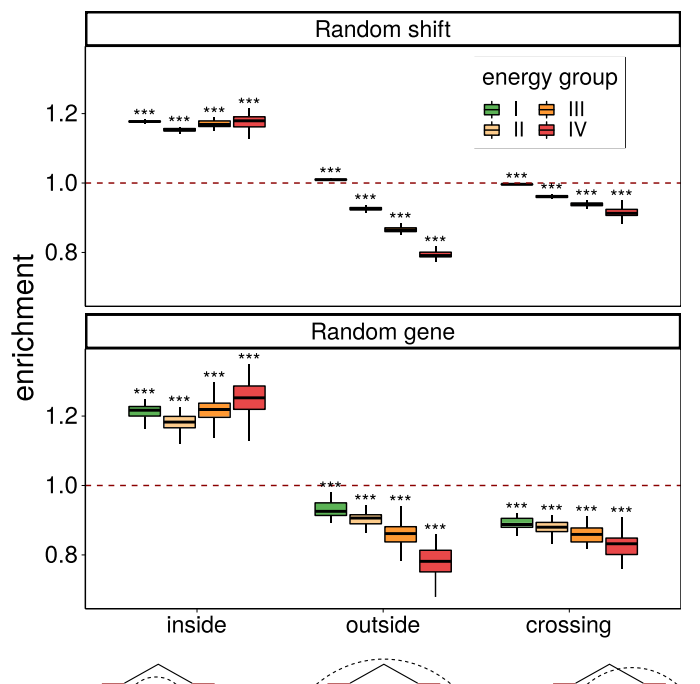

D

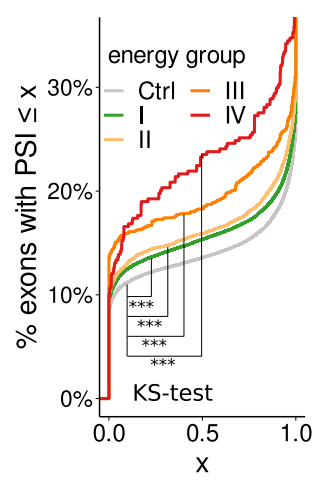

Introns

C

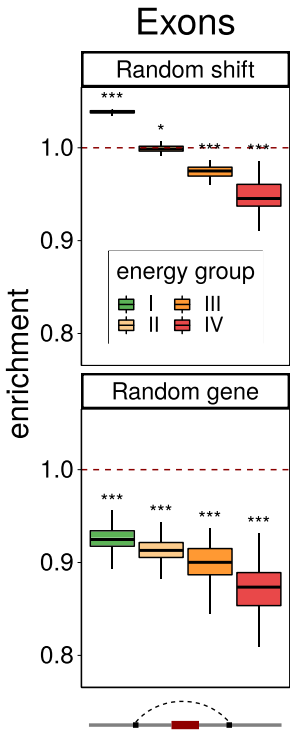

$\mathbf{E}$

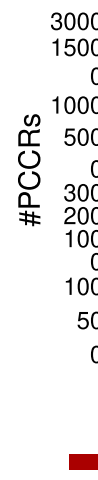

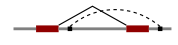
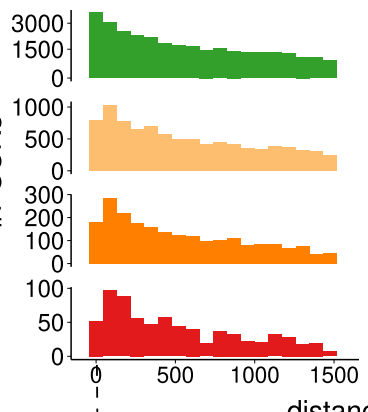

distance, nt
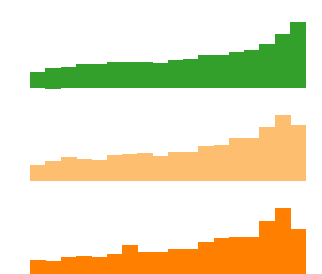

Fig. 3 Splicing. A Control procedures. In the random shift control, a PCCR is shifted within the gene. In the random gene control, a pseudo-PCCR is created in the same relative position of a different gene chosen at random. The number of PCCRs inside, outside, and crossing the reference set of intervals (e.g., introns) are counted. B PCCRs are enriched inside introns and depleted in outside and crossing configurations. C PCCRs looping out exons are depleted. D The cumulative distribution of the average exon inclusion rate $(\Psi)$ in HepG2 cell line for exons looped out by PCCRs of the four energy groups vs. exons not looped out by PCCRs (Ctrl). KS denotes the two-sample Kolmogorov-Smirnov test. Sample sizes for energy groups I-IV and control are $n=$ 73,366, 13,974, 1877, 374, and 161,947, respectively. E The distribution of distances from intronic PCCRs to intron ends (bin size 75 nts). Group I PCCRs are enriched, while group IV PCCRs are depleted in 75-nt windows immediately adjacent to splice sites. In all panels, boxplots (represented by the median, upper and lower quartiles, upper and lower fences; outliers are not shown) correspond to $n=40$ randomizations; ${ }^{\star}$ and ${ }^{\star \star \star}$ denote a statistically discernible difference at the $5 \%$ and $0.1 \%$ significance level, respectively (in panels $\mathbf{B}$ and $\mathbf{C}$ for a two-tailed Wilcoxon's test with $H_{0}$ : enrichment $=1$ ).

be counted more than once if it is located inside one intron and crosses another). In the first control set, referred to as random shift, each PCCR was shifted randomly within its gene. The resulting pseudo-PCCR had the same spread and belonged to the same gene as the original PCCR. In the second control set, referred to as random gene, for each gene and a PCCR in it, we created a pseudo-PCCR at the same relative position as the original PCCR, but in a randomly chosen gene of the same length. The resulting pseudo-PCCR had the same spread and the same relative position as the original PCCR, but belonged to a different gene. Repeated sampling of these sets allowed estimation of statistical significance.

In comparison with the set of annotated introns by random shifts, PCCRs showed a pronounced enrichment in the inside category and depletion in the outside and crossing categories, with the magnitude of depletion increasing for more stable structures (Fig. 3B, top). To rule out a possibility that preferential PCCR positioning within introns originates from uneven distribution of longer introns along the gene, with $5^{\prime}$ introns being on average longer ${ }^{27,28}$, we repeated the same analysis using 
random gene control, and the enrichment and depletion remained significant (Fig. 3B, bottom). A similar comparison with the set of annotated exons revealed a substantial depletion of PCCRs that loop out exons, which also became stronger as PCCR stability increased (Fig. 3C). The average inclusion rate of exons that were looped out by PCCR was lower than that of exons that are not surrounded by PCCR, with the magnitude of the difference increasing for more stable structures (Fig. 3D). These results reconfirm that PCCRs generally tend to avoid placing exons in a loop, which promotes exon skipping.

The frequency of intronic PCCRs decays with increasing the distance to intron ends in all four energy groups partially reflecting the decrease of sequence conservation (Fig. 3E). However, weaker structures occur more frequently in $75-\mathrm{nt}$ windows adjacent to splice sites, while stronger structures tend to occupy more distant positions. The ends of PCCRs tend to be closer to exon boundaries than would be expected by chance from random shifts (Fig. S8). Contrary to what was reported earlier for Drosophila melanogaster, there is no substantial depletion of CCR in polypyrimidine tracts (PPTs). This indicates a large regulatory potential for splicing since a large fraction of CCR that overlaps PPT (43.2\%) also blocks the acceptor splice site. In addition, there were on average $20 \%$ fewer overlaps of CCR with intronic branch points ${ }^{42}$ than would be expected from random shift control, that is, CCRs tend not to overlap intronic branch points.

To test whether PCCRs interfere with splicing signals, we identified intronic motifs with high similarity to donor and acceptor site consensus sequences (cryptic splice sites) and analyzed the expression of splice sites in a large compendium of RNA-sequencing (RNA-seq) samples from the Genotype Tissue Expression Project (GTEx) ${ }^{43}$. CCRs overlapping highly expressed (active) splice sites were depleted, while CCRs overlapping splice sites with low read support (inactive) were enriched (Fig. 4A). CCRs overlapping non-expressed cryptic splice sites were also enriched with the exception of highly stable structures, which are likely devoid of cryptic splice sites due to high GC content. We also found that the largest enrichment of CCRs overlapping candidate cryptic splice sites was among PCCR with small spread (MW test, $p$ value $=0.004$ ), in agreement with the previous findings that cryptic splice sites tend to be suppressed by local RNA secondary structure ${ }^{44,45}$.

RNA secondary structure has been suggested to be a defining feature that leads to backsplicing and formation of circular RNAs (circRNA) ${ }^{46}$. A comparison with the set of circRNAs from the tissue-specific circRNA database (TCSD) ${ }^{47}$ by random shift and random gene controls revealed a pattern opposite to that of linear introns, in which PCCRs were enriched outside circRNAs and depleted inside circRNAs (Fig. 4B). This supports the hypothesis that circRNAs originate from loop-out sequences that are formed by stable double-stranded RNA structures ${ }^{46}$.

RNA editing. A widespread form of post-transcriptional RNA modification is the enzymatic conversion of adenosine nucleosides to inosine, a process called A-to-I editing, which is mediated by the adenosine deaminase acting on RNA (ADAR) family of enzymes ${ }^{48,49}$. Since ADAR editing occurs in double-stranded RNA substrates ${ }^{50,51}$, we asked whether A-to-I editing sites are enriched among CCRs (Fig. 4C). We observed a strong enrichment of ADAR-edited sites that are documented in the RADAR database $(2,576,459$ sites) within CCR as compared to non-CCR parts of CIRs (odds ratio $(\mathrm{OR})=2.1 \pm 0.2$ ). While the OR was nearly the same for PCCR with energy from -15 to $-30 \mathrm{kcal} / \mathrm{mol}$, it dramatically increased for PCCR with the energy $<-30 \mathrm{kcal} / \mathrm{mol}(2.1 \pm 0.2$ vs. $13.2 \pm 5.0)$, reconfirming previous observations that ADAR targets are long double-stranded
$\mathrm{RNAs}^{52}$. The OR was also significantly greater $(1.7 \pm 0.2$ vs. $22.2 \pm 13.1)$ for PCCR with higher evidence of compensatory mutations, but it did not depend significantly on the PCCR spread. These results were concordant with each other for the datasets of A-to-I editing sites from RADAR and REDIportal databases $^{53,54}$ and can be regarded as an additional support for double-stranded structure of PCCRs.

$5^{\prime}$-end and $3^{\prime}$-end processing. Recent reports indicate that RNA structure is important for the $3^{\prime}$-end processing of human mRNAs ${ }^{8}$, and that competing RNA base pairings could be involved in alternative splicing and polyadenylation in the $3^{\prime}$-variable region ${ }^{55}$. In this section, we characterize the relationship between PCCRs, $5^{\prime}$-end, and $3^{\prime}$-end mRNA processing using the data from transcript annotation databases ${ }^{56}$ and clusters of poly(A)-seq and cap analysis of gene expression (CAGE) tags ${ }^{57,58}$

First, we estimated the number of transcripts that start or end within CCRs, including all incomplete and aberrant isoforms that are annotated in GENCODE (Fig. 4D). Both $5^{\prime}-$ and $3^{\prime}$ ends were enriched inside CCRs, suggesting that double-stranded structures are associated with the suppression of aberrant transcripts. Next, we asked whether the annotated $5^{\prime}$ and $3^{\prime}$ ends of transcripts were enriched in the inner part of PCCRs, that is, in the regions between paired CCRs. Indeed, both were significantly enriched, and the magnitude of the enrichment increased with increasing PCCR stability (Fig. 4E). The random gene control confirmed that the effect was not due to nonuniform distribution of PCCRs. To rule out the possibility that non-expressed isoforms contribute to the observed enrichment, we additionally examined the expressed CAGE tags and poly(A)-seq clusters and found that they were also significantly enriched within PCCR (Fig. S9). Since PCCRs are, in turn, enriched inside introns, this motivated us to analyze triple associations between RNA structure, splicing, and end processing.

Thus, we asked whether the annotated transcript ends, CAGE tags, and poly(A)-seq clusters occur more frequently in introns that contain PCCRs. However, introns with PCCRs tend to be also longer than introns without PCCRs, which may affect the above frequencies. Hence, we subdivided all annotated introns into two groups, introns with PCCR (IWP) and introns without PCCR (IWO), and selected two samples from IWP and IWO, 38,119 introns each, with matching intron lengths (Fig. S10). Of these, 12,782 (33.5\%) IWP contained at least one annotated $3^{\prime}$ end, while only $9,575(25.1 \%)$ IWO did so (OR $=1.50 \pm 0.05)$. Similarly, 12,042 (31.6\%) IWP contained at least one annotated $5^{\prime}$ end, compared to $8,978(23.6 \%)$ for IWO (OR $=1.50 \pm 0.05)$. The enrichment of both $5^{\prime}$ and $3^{\prime}$ ends in IWP raises an intriguing hypothesis that there could be an RNA structure-mediated coupling between splicing and end processing (see "Discussion").

Mutations. Mutations generally lower the stability of RNA secondary structure, and some of them are under evolutionary selection owing to their effects on the thermodynamic stability of pre-mRNA ${ }^{59}$. In order to estimate the impact of human population polymorphisms on PCCRs, we analyzed single-nucleotide polymorphisms (SNPs) from the 1000 Genomes project ${ }^{60}$ and compared SNP density in CCR with that in the remaining parts of CIR. Germline SNPs were significantly underrepresented in CCR (0.0196 vs. 0.0207 SNPs per nt, one-tailed Poisson test, $p$ value $<$ 0.01). Next, for each SNP that occurs in a PCCR, we calculated the free energy change caused by the mutation and compared it to the free energy change that would have been observed if the same mutation occurred at a different position of the same CCR (Fig. S11A). It turned out that actual SNPs destabilized PCCRs less than it would be expected by chance (Wilcoxon's test, 
A

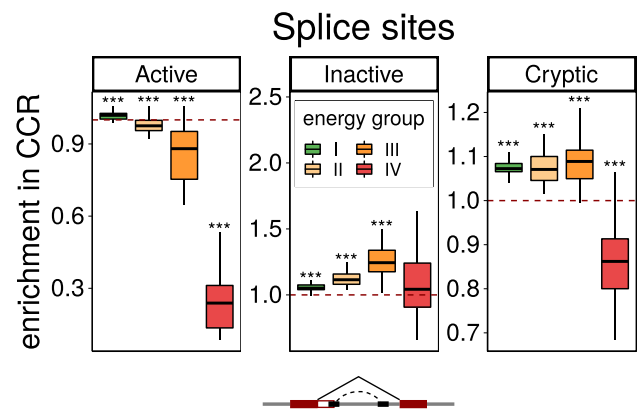

C

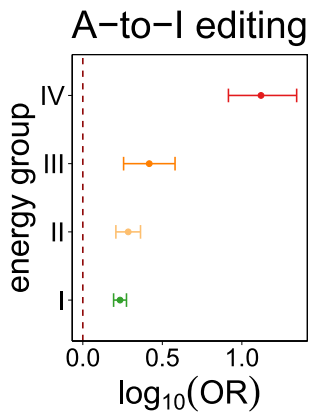

B

Circular RNA

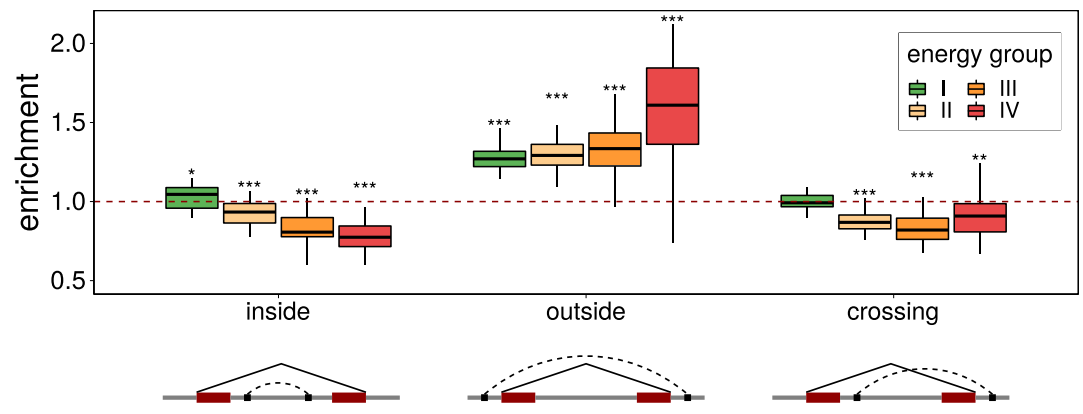

D

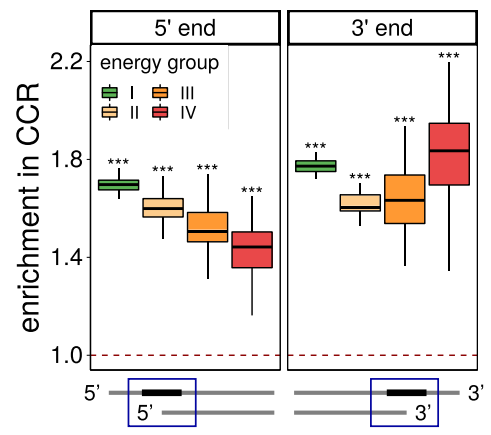

$\mathbf{E}$

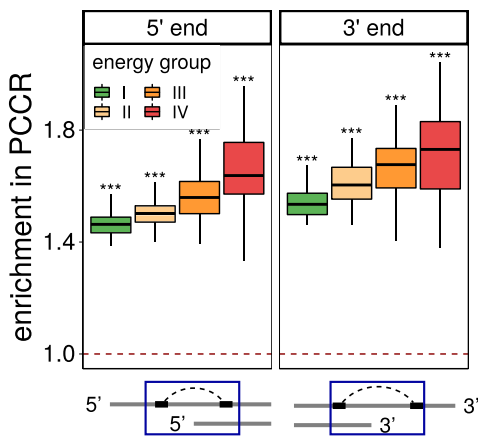

Fig. 4 Splicing, RNA editing, and end processing. A CCRs are depleted around actively expressed splice sites and enriched around inactive and cryptic splice sites. B PCCRs are enriched outside back-spliced introns (circular RNAs from TCSD, ref. ${ }^{47}$ ) and depleted in inside and crossing configurations. C CCRs are enriched with A-to-I RNA-editing sites (RADAR REDIportal53,54); OR denotes the odds ratio (see "Methods"); error bars represent the 95\% confidence intervals. D CCRs are enriched with $5^{\prime}$ and $3^{\prime}$ ends of transcripts annotated in GENCODE database (including all aberrant and incomplete transcripts). That is, transcript ends frequently occur in double-stranded parts of PCCRs. E PCCRs are also strongly enriched with $5^{\prime}$ and $3^{\prime}$ ends of transcripts, that is, the annotated transcript ends frequently occur in the loop between double-stranded parts of PCCRs. In all panels, boxplots (represented by the median, upper and lower quartiles, upper and lower fences; outliers are not shown) correspond to $n=40$ randomizations; ${ }^{\star},{ }^{\star}$, and ${ }^{\star \star \star}$ denote a statistically discernible difference at the $5 \%, 1 \%$, and $0.1 \%$ significance level, respectively, for a two-tailed Wilcoxon's test with $H_{0}$ : enrichment $=1$.

$p$ value $<0.001)$, suggesting that SNPs generally tend to minimize their destabilizing impact on RNA structure.

Compensatory mutations may also occur in the human population, but at a substantially lower frequency. To estimate the frequency of compensatory population polymorphisms, we identified PCCRs that contain SNPs with an allele frequency of $1 \%$ and higher. Out of 64,074 bp in PCCRs that were affected by SNPs, only 12 showed sufficient support for compensatory mutations. After randomization, in which the pairs of complementary nucleotides were randomly switched, the respective average values were 64,132 and 0.06 (see "Methods," Fig. S11B). The density of compensatory mutations normalized to the number of base pairs with mutations were $0.019 \%$ for the actual base pairs and $(9.0 \pm 7.3) \times 10^{-5} \%$ for the randomized set. From this we conclude that, despite compensatory mutations within PCCR are quite rare in the human population, they are significantly enriched (one-tailed Poisson's test, $P<0.01$ ).
RBP binding sites. Multiple lines of evidence indicate that binding of RNA-binding proteins (RBPs), which is crucial for coand post-transcriptional RNA processing, depends on RNA structural context ${ }^{61-63}$. To assess the association between longrange RNA structure represented by PCCRs and RBP binding, we computed eCLIP peak frequencies ${ }^{64,65}$ in the vicinity of CCRs and compared them to the background eCLIP peak frequencies in CIRs surrounding CCRs. Among factors that showed a substantial enrichment, there were RBPs that are known to exert their function in conjunction with RNA structure such as $R B F O X 2^{32}$ and factors that favor increased structure over their motifs such as SRSF9 and SFPQ ${ }^{66,67}$ (Fig. 5A). For RBPs with single-stranded RNA-binding activity such as ILF3 and $h n R N P A 1$, we observed a significant depletion of binding sites within CCRs (Fig. S12) ${ }^{68,69}$.

One of the features of the eCLIP protocol is that an RBP can crosslink with either RNA strand that is adjacent to the 
$\mathbf{A}$
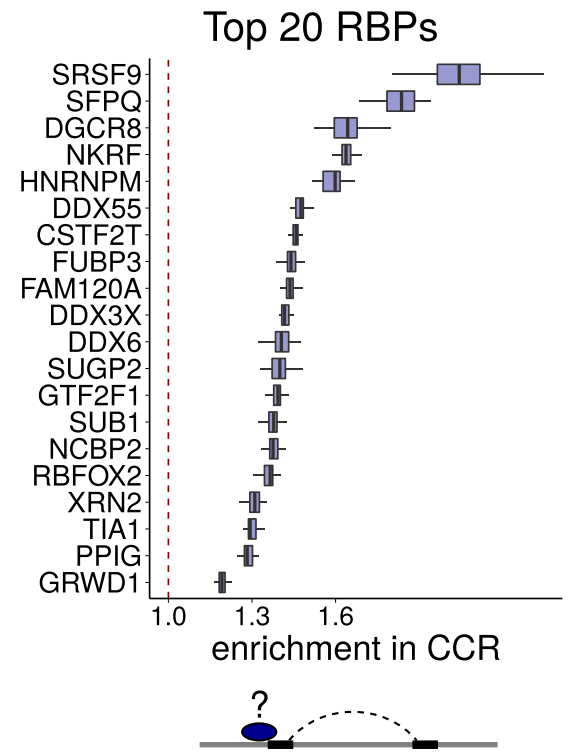

C

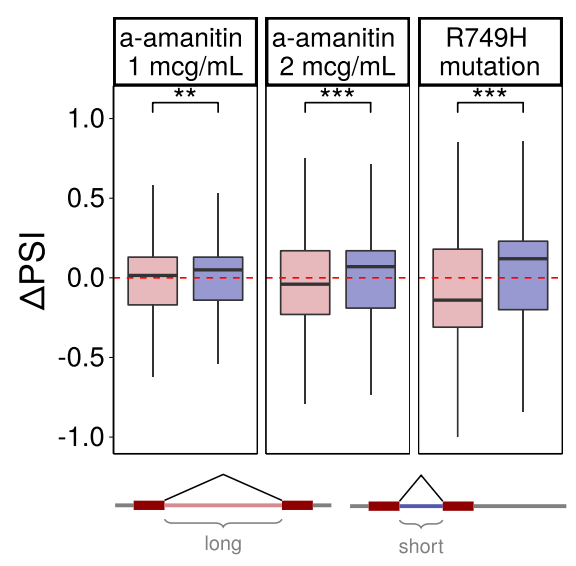

B

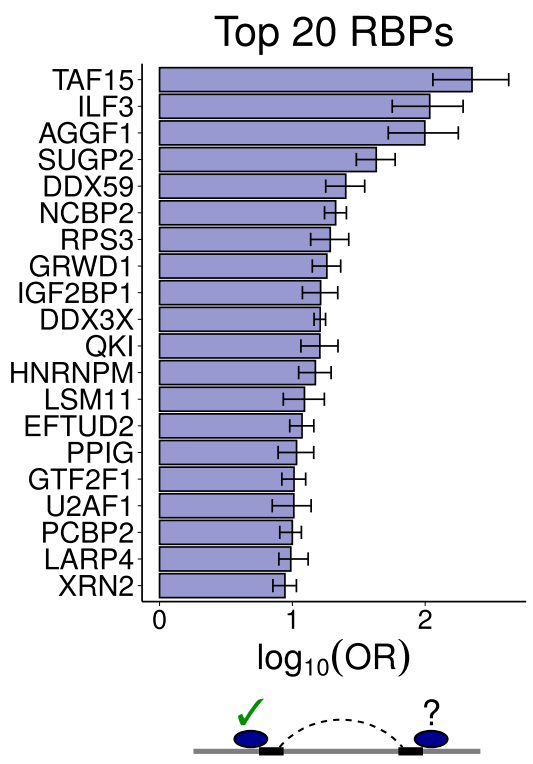

D

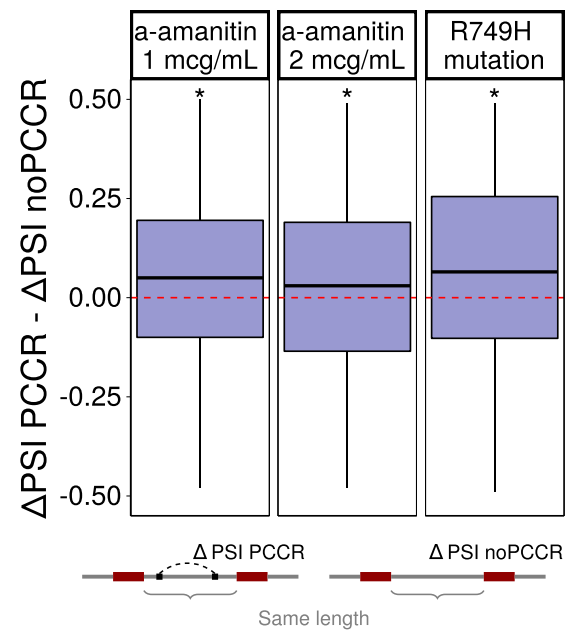

Fig. 5 RNA-binding proteins (RBP). A According to eCLIP profiles, CCRs are enriched within binding sites of some RBPs (top 20 RBPs are shown). The RBPs that show depletion of CCRs are listed in Fig. S12. Boxplots represent $n=40$ random shifts of CCR within CIR. B The odds ratios (OR) of RBP binding near both CCR in PCCR given that RBP binds near at least one CCR indicate that PCCRs are enriched with forked eCLIP peaks. Error bars represent the $95 \%$ confidence intervals. C The change of inclusion rate $(\Delta \Psi)$ of exons following short introns $(n=2844,2650$, and $4032 \mathrm{for} 1 \mu \mathrm{g} / \mathrm{mL}, 2 \mu \mathrm{g} / \mathrm{mL}$, and R749H mutant, respectively) vs. exons following long introns ( $n=2931,2762$, and 3807 for $1 \mu \mathrm{g} / \mathrm{mL}, 2 \mu \mathrm{g} / \mathrm{mL}$, and R749H mutant, respectively) in response to RNA Pol II slowdown with $\alpha$-amanitin and in the slow RNA Pol II mutant R749H ${ }^{71}$. D The difference between the inclusion rate change of exons following introns with a PCCR $(\triangle \Psi P C C R)$ and the inclusion rate change of exons following introns of the same length, but without PCCRs ( $\triangle \Psi$ InoPCCR) in response to RNA Pol II slowdown ( $n=191,184$, and 156 for $1 \mu \mathrm{g} / \mathrm{mL}, 2 \mu \mathrm{g} / \mathrm{mL}$, and R749H mutant, respectively). In all panels, boxplots are represented by the median, upper and lower quartiles, upper and lower fences without outliers; ${ }^{\star},{ }^{\star *}$, and ${ }^{\star \star \star}$ denote a statistically discernible difference at the $5 \%, 1 \%$, and $0.1 \%$ significance level, respectively (two-tailed Mann-Whitney and Wilcoxon's tests, in panel $\mathbf{D}$ with respect to $H_{0}: \Delta \Psi P C C R-\Delta \Psi$ noPCCR $=0$ ).

double-stranded region. Hence, we expect a higher chance of observing an eCLIP peak near the CCR given that the other CCR in the pair contains a nearby peak, a situation that will be referred to as forked eCLIP peak. To estimate the magnitude of this association, we computed the respective OR and found that the vast majority of RBPs ( 64 out of 74, $p$ value $<10^{-12}$ ) indeed have a substantially higher likelihood of binding close to a CCR given that they bind the other CCR in a pair (Fig. 5B). This can be regarded as independent evidence for double-stranded structure of PCCRs. Interestingly, the largest OR was observed for TAF15, a TBP-associated factor 15, which is not a double-stranded RNA (dsRNA)-binding protein itself, but interacts with FUS, which is capable of binding dsRNA ${ }^{70}$. Similarly, ILF3, which showed a depleted RNA binding within CCRs, nevertheless is positively associated with forked eCLIP peaks, suggesting its binding at single-stranded regions adjacent to PCCRs. This indicates, on the one hand, that RBP binding is inseparable from the surrounding RNA structure and, on the other hand, that eCLIP peaks may not correctly reflect the actual binding positions of RBPs since they are affected by intramolecular base pairings and interactions with other players.

RNA Pol II elongation speed. The kinetic model of cotranscriptional splicing suggests that RNA Pol II elongation slowdown expands the "window of opportunity" for the recognition of weak splice sites, thereby increasing the rate of inclusion of upstream exons ${ }^{71,72}$. Besides this direct impact on splice site recognition, slow RNA Pol II elongation may also affect the way the transcript folds, which is another important determinant of 
how the transcript will be processed by the splicing machinery ${ }^{73}$. To investigate the role of long-range RNA structure in cotranscriptional splicing, we performed RNA-seq experiments, in which we used $\alpha$-amanitin to slow down the RNA Pol II elongation speed ${ }^{74}$, and additionally analyzed publicly available data on the impact of RNA Pol II elongation speed on splicing ${ }^{71}$.

The expected consequence of the RNA Pol II slowdown is that the inclusion rate of exons that follow short introns will increase, and the inclusion rate of exons that follow long introns will decrease. Indeed, this trend was observed both when RNA Pol II elongation speed was decreased by $\alpha$-amanitin and in the slow RNA Pol II mutant R749H (Fig. 5C) ${ }^{71}$. To check whether RNA Pol II slowdown differently affects introns with and without PCCRs, we matched each exon that follows an intron containing a PCCR with a randomly chosen exon that follows an intron of the same length, but without PCCRs. The difference in inclusion rates of these matched exons showed that exons that follow an intron with a PCCR tend to be more included than exons following an intron without PCCRs at both concentrations of the inhibitor and in R749H RNA Pol II mutant (Fig. 5D). This can be considered as evidence for RNA Pol II slowdown to affect exon inclusion through pre-mRNA folding, in addition to modulation of splice site recognition. Namely, slower RNA Pol II elongation speed may not only facilitate the processing of upstream splice sites by the spliceosome but also allow sufficient time for the intronic RNA structure to fold, thus promoting exon inclusion. A particular example of such a kinetic mechanism linked to RNA structure was reported for the Ate1 gene, in which a long-range base pairing dynamically regulates the ratio of mutually exclusive exons $^{36}$.

Case studies. In this section, we focus on the association of longrange base pairings with RBP binding in the context of two particular splicing-related mechanisms, RNA bridges ${ }^{32}$, and exon loop-outs ${ }^{33}$.

To identify potential RNA bridges, that is, long-range RNA structures that bring an RBP binding site closer to the regulated exon $^{32}$, we searched for candidate binding sites of RBPs profiled by eCLIP that were located within 50 nts from a CCR, on the one hand, and exons located within 50 nts from its mate CCR, on the other hand. To detect regulation, we additionally required that exon inclusion rate significantly respond to shRNA knockdown (shRNA$\mathrm{KD}$ ) of the same factor using the data on RBP KDs produced by the ENCODE Consortium (see "Methods") ${ }^{65}$. This procedure yielded a set of 296 candidate RNA bridges (Supplementary Data File 3), including the RNA bridge that controls the inclusion of exon 12 of ENAH gene (Fig. 6A). A PCCR with the hybridization energy $-19.8 \mathrm{kcal} / \mathrm{mol}$ coincides with the core part of the RNA stem that was reported earlier ${ }^{32}$. We reconfirm that it is surrounded by forked eCLIP peaks of RBFOX2, which reflects cross-linking next to the double-stranded region, and that the inclusion of exon 12 drops by $43 \%(\triangle \Psi=-0.43)$ upon $R B F O X 2 \mathrm{KD}$. However, we also find a nested PCCR with the hybridization energy $-20.4 \mathrm{kcal} / \mathrm{mol}$, which suggests that the RNA bridge extends much further than it was reported originally. As a novel example, we describe a candidate RNA bridge in the $3^{\prime}$ end of RALGAPA1 gene, which encodes the major subunit of the RAL-GTPase-activating protein ${ }^{75}$. In this gene, a group of nested PCCRs approximates binding sites of RBFOX2 and $Q K I$ to the penultimate exon (Fig. 6B). The KD of each of these factors promotes exon skipping, which indicates that exon inclusion depends on the binding of RBFOX2 and QKI through an RNA bridge.

In a similar way, we identified candidate base pairings that loop out exons by searching for PCCRs that surround an exon and contain an RBP binding site within one of the CCRs. In addition, we required that the exon significantly respond to RBP KD. This procedure yielded a set of 1135 candidate exon loop-outs (Supplementary Data File 4). Among them, there were two nested RNA structures looping out exon 24 of GPR126, the human $G$ protein-coupled receptor 126, in which one of the CCRs overlaps a RBFOX2 binding site, and the exon responds to RBFOX2 KD (Fig. 6C). Another example is the alternative $3^{\prime}$-end exon in FGFR1OP2, fibroblast growth factor receptor 1 oncogene partner 2, which is suppressed by $Q K I \mathrm{KD}$ and, at the same time, is looped out by a PCCR that overlaps a $Q K I$ binding site (Fig. 6D).

Data visualization and availability. The tables listing PCCRs for GRCh37 and GRCh38 human genome assemblies are available in BED format as Supplementary Data Files 1 and 2, respectively. These predictions are visualized through a track hub for the UCSC Genome Browser ${ }^{31}$. In order to connect the track hub, the following link https://raw.githubusercontent.com/kalmSveta/ PCCR/master/hub.txt can be copied and pasted into the form https://genome.ucsc.edu/cgi-bin/hgHubConnect\#unlistedHubs. The PCCR tracks are grouped by the energy groups, spread, and $E$ value. Along with PCCRs, we additionally report the response of exons to RNA Pol II elongation slowdown, icSHAPE reactivity scores, and RIC-seq predictions. The tables listing the predicted RNA bridges and looping-out PCCRs are available as Supplementary Data Files 3 and 4, respectively. They are visualized through https://raw.githubusercontent.com/kalmSveta/ RNA-bridges/master/hub.txt, along with eCLIP data and exon responses to shRNA KDs. Supplementary Data Files are available online through ZENODO repository https://zenodo.org/record/ 4603132 (also see https://doi.org/10.5281/zenodo.4603132) and via http://arkuda.skoltech.ru/ dp/shared/PrePH/.

\section{Discussion}

It has been increasingly acknowledged that RNA structure plays a critical role in the regulation of eukaryotic gene expression at all steps from transcription to translation, but little attention has been paid to long-range RNA structure. From the thermodynamic standpoint, long-range base pairings contribute to the enthalpy of RNA folding as much as local base pairings do, and the corresponding energy figures exceed by an order of magnitude the typical folding energies of globular protein domains ${ }^{76}$. However, since RNA structure affects, and is itself strongly affected by RBP binding, a reliable prediction of long-range RNA structure in full-length eukaryotic transcripts does not seem feasible at the current state of the art. Instead of the detailed structure, here we consider as a proxy for RNA structure the core of highly stable and evolutionarily conserved double-stranded regions, different combinations of which may represent one or several physiologically relevant folds.

The existence of associations between long-range RNA structure and splicing has been noted in the previous studies ${ }^{7}$, including our earlier reports ${ }^{27-29}$. The trends that were proposed for smaller sets also hold for the extended catalog of PCCRs presented here, namely, the preference of PCCRs to be positioned within introns proximally to splice sites ${ }^{27}$, lower inclusion rate of looped out exons ${ }^{33,77}$, circumscription of circRNAs ${ }^{78}$, avoidance of intronic branch points ${ }^{79,80}$, and generally obstructive effect on splice sites that are implicated in double-stranded structure ${ }^{81-83}$. We additionally observed a remarkable overlap of PCCRs with A-to-I RNAediting sites and multiple associations with forked eCLIP peaks, which reveal traces of multimolecular complexes with patterns that are specific to double-stranded regions. These findings reconfirm the well-known mechanism of ADAR-mediated pathway ${ }^{84}$ and show the importance of RNA structure for the assembly of 
A

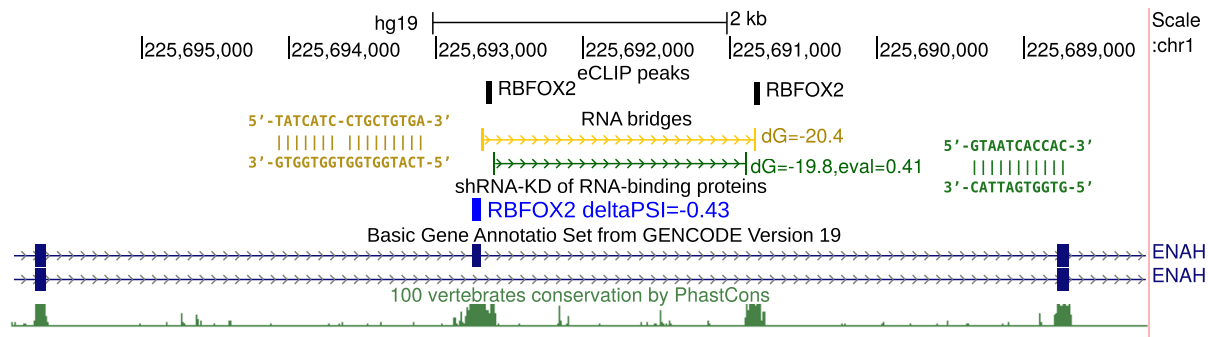

B

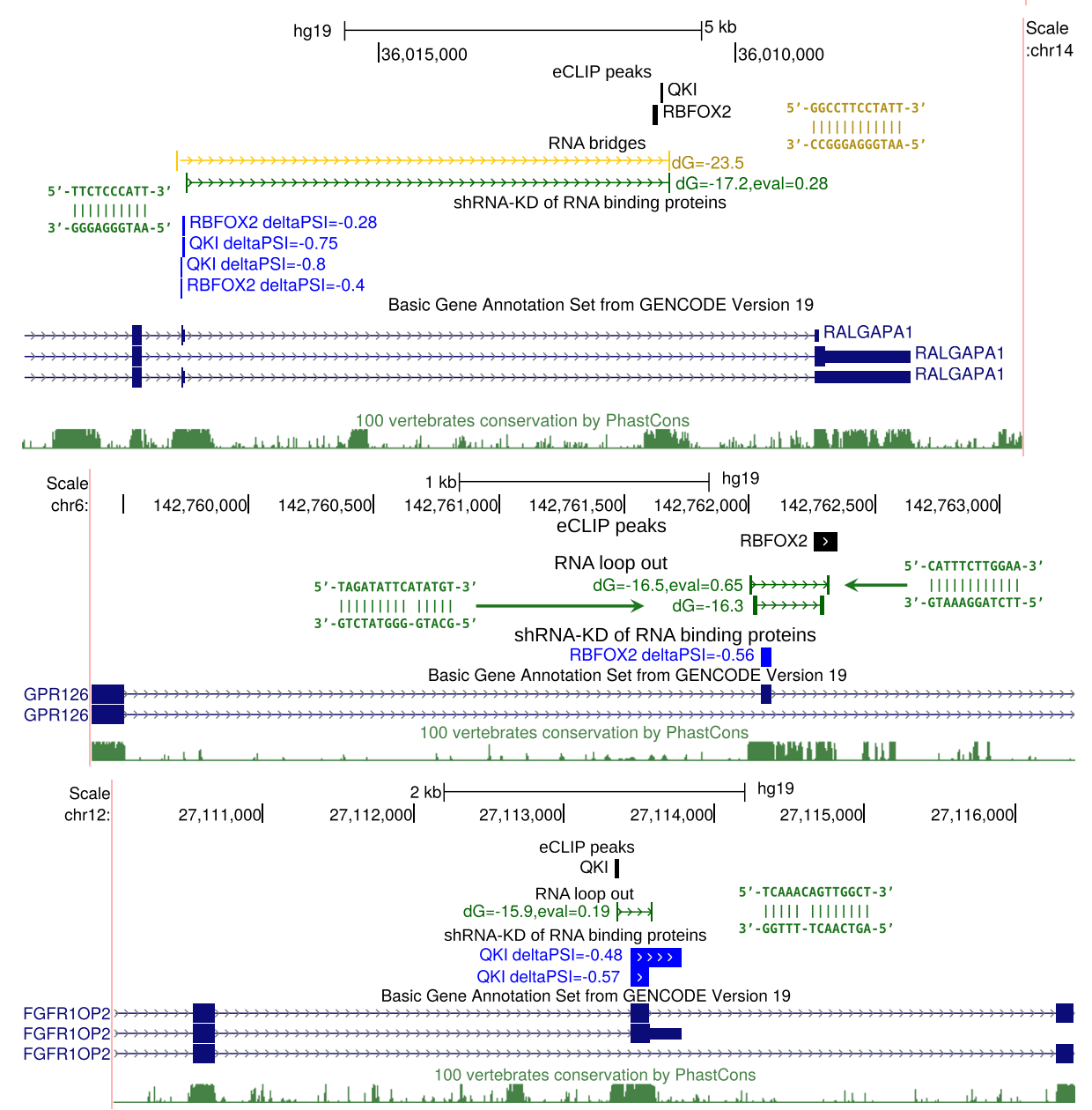

Fig. 6 Case studies. A An RNA bridge in ENAH gene brings a distant RBFOX2 binding site into proximity of the regulated cassette exon ${ }^{32}$. The exon inclusion rate substantially decreases under RBFOX2 depletion $(\triangle \Psi=-0.43)$. B The predicted RNA bridge in RALGAPA1 brings distant binding sites of $R B F O X 2$ and $Q K I$ to the regulated exon. The exon significantly responds to the depletion of these two factors $(\Delta \Psi=-0.28$ and $\Delta \Psi=-0.75$, respectively). C A cassette exon in GPR126 is looped out by a PCCR overlapping an eCLIP peak of RBFOX2 and significantly responds to $R B F O X 2$ depletion $(\triangle \Psi=-0.56)$. D An alternative terminal exon in FGFR1OP2 is looped out by a PCCR overlapping an eCLIP peak of $Q K I$ and significantly responds to $Q K I$ depletion $(\triangle \Psi=$ -0.48 ). In all panels, exon inclusion rate changes are statistically significant ( $q$ value $<0.01$ ).

RNA-protein complexes, with a preference for some RBPs and avoidance for the others ${ }^{65,66}$. At the same time, they may also be regarded as independent support for double-stranded structure of PCCRs in addition to the evidence from experimental RNA structure profiling and compensatory substitutions.

The components of RNA-processing machinery operate in a strict coordination not only in space but also in time. The kinetic profile of RNA Pol II elongation has a significant impact on alternative splicing ${ }^{85}$. Slow RNA Pol II elongation generally opens a window of opportunity for weak splice sites to be recognized, leading to higher inclusion of alternative exons, although in some cases the effect can be quite opposite ${ }^{86,87}$. Slow RNA Pol II elongation may also influence poly(A) site choice by enhancing the recognition of suboptimal polyadenylation signals (PASs) ${ }^{88}$ Consistent with this, we observe an increased inclusion of exons that are preceded by shorter introns. However, we also observe an additional component to this general trend, one in which structured and unstructured RNAs respond differently to RNA Pol II slowdown. This observation indicates that long-range RNA structure could coordinate the interaction between spatial and temporal components of splicing regulation. The example of long-range RNA structure in the Ate1 gene demonstrates that mechanisms similar to bacterial attenuation may also take place in eukaryotic cells $36,89,90$.

RNA structure is implicated in the recognition of PASs by cleavage and polyadenylation specificity factor (CPSF) and 
facilitates the $3^{\prime}$-end processing by juxtaposing PAS and cleavage sites that are otherwise too far apart ${ }^{8,91}$. Functional RNA structures in $5^{\prime}$-untranslated regions are also implicated in the regulation of translation ${ }^{11}$. While these reports mostly concern local RNA structure, an intriguing finding of this work is the link between long-range RNA structure and pre-mRNA $3^{\prime}$-end processing, which is manifested by the enrichment of poly $(\mathrm{A})$-seq clusters in the inner part of PCCRs. It indicates that mechanisms other than sequestration or spatial convergence of PAS and cleavage sites may be involved ${ }^{92,93}$. In fact, human genes contain thousands of dormant intronic PASs that are suppressed, at least in part, by $\mathrm{U} 1$ small nuclear ribonucleoproteins in a process called telescripting ${ }^{94}$. While the exact mechanism of this suppression is not known, many intronic PASs were found to be associated with CstF64, a ubiquitous pre-mRNA $3^{\prime}$-processing factor ${ }^{95}$, suggesting that cleavage and polyadenylation machinery may actually operate in all introns constitutively. Could it be that RNA structure helps to suppress premature intronic polyadenylation?

Figure 7 illustrates a hypothetical mechanism of suppression of intronic polyadenylation by co-transcriptional splicing, which explains the enrichment of poly(A)-seq clusters in the inner parts of PCCRs. Indeed, the cleavage and polyadenylation of a structured pre-mRNA could be rescued by co-transcriptional excision of the intron, while RNA structure stabilizes the molecule through intramolecular base pairings despite the disruption of the backbone (Fig. 7A). However, such a rescue would not happen in unstructured RNAs when splicing has a delay relative to cleavage and polyadenylation (Fig. 7B). This scenario is further supported by a conspicuous association between transcript $5^{\prime}$ and $3^{\prime}$ ends in exhaustive transcriptome annotations, a typical example of which is an intron that contains a $3^{\prime}$ end of protein-coding transcript and a $5^{\prime}$ end of another, usually noncoding transcript (Fig. S7). It is $2.86 \pm 0.10$ times more likely to see a $5^{\prime}$ end in an intron that contains a $3^{\prime}$ end, and hence the enrichment of poly(A)-seq clusters within PCCRs also implies the enrichment of $5^{\prime}$ ends and CAGE clusters. The described mechanism could be responsible for the generation of transcripts with alternative $3^{\prime}$ ends, for example, for the RNA structure-mediated switch between splicing and polyadenylation in the Nmnat gene in D. melanogaster ${ }^{27}$.

RNA structure probing by icSHAPE and the assessment of long-range RNA-RNA interactions by photo-inducible RNA cross-linking are the most current techniques for global analysis of RNA structure in vivo ${ }^{18,22-24}$. However, these data reflect gene expression patterns that are specific to cell lines, in which they were generated, and have strong undercoverage bias in intronic regions, which are spliced out and degraded. The reduction of the intronic signal is also a common problem for RNA cross-linking and immunoprecipitation experiments ${ }^{96}$. In addition, it was meaningful to compare PCCRs only to intramolecular RNA contacts that belong to CIRs and do not exceed the distance limit of 10,000 nts. Consequently, the validation with respect to these assays was possible only for a small number of PCCRs, on which the comparison, however, showed a concordant result (Table 2). On the other hand, the fact that 2961 out of 916,360 PCCRs were supported by RIC-seq is highly significant compared to the expected intersection of 381 structures for two interval sets of the same size obtained by random shifts.

The major problem of the current method is the high number of false-positive predictions. On the one hand, the procedure for the estimation of FDR is based on the assumption that premRNAs of different genes do not interact with each other, and that regulatory sequences in different genes evolve independently. However, psoralen cross-linking and proximity RNA ligation assays have demonstrated that this assumption may not be completely true because RNA-RNA interactions in trans are highly abundant ${ }^{20-23}$. Therefore, the re-wiring control overestimates FDR, as it did in previous works ${ }^{29}$. On the other hand, the amount of random complementarity in CIRs is, indeed, quite large. For instance, two intronic binding sites of a transcription factor that occur on opposite DNA strands will be detected as a PCCR, and they may even be supported by spurious compensatory substitutions that result not from selective constraints on RNA structure, but from evolving specificity of the binding site. An example of this is the RP11-439A17.4 lncRNA, a part of which is complementary to conserved sequences in 22 mammalian histone genes, but the complementary elements are, in fact, the binding sites of MEF-2A, a myocyte-specific transcription factor $^{29}$. Evolution maintains them conserved and technically complementary for reasons other than base pairing, which makes this situation in principle indistinguishable from evolutionary selection acting on true RNA structures. To reduce FDR, a significant improvement could be achieved by combining the methodology presented here with the emerging experimental

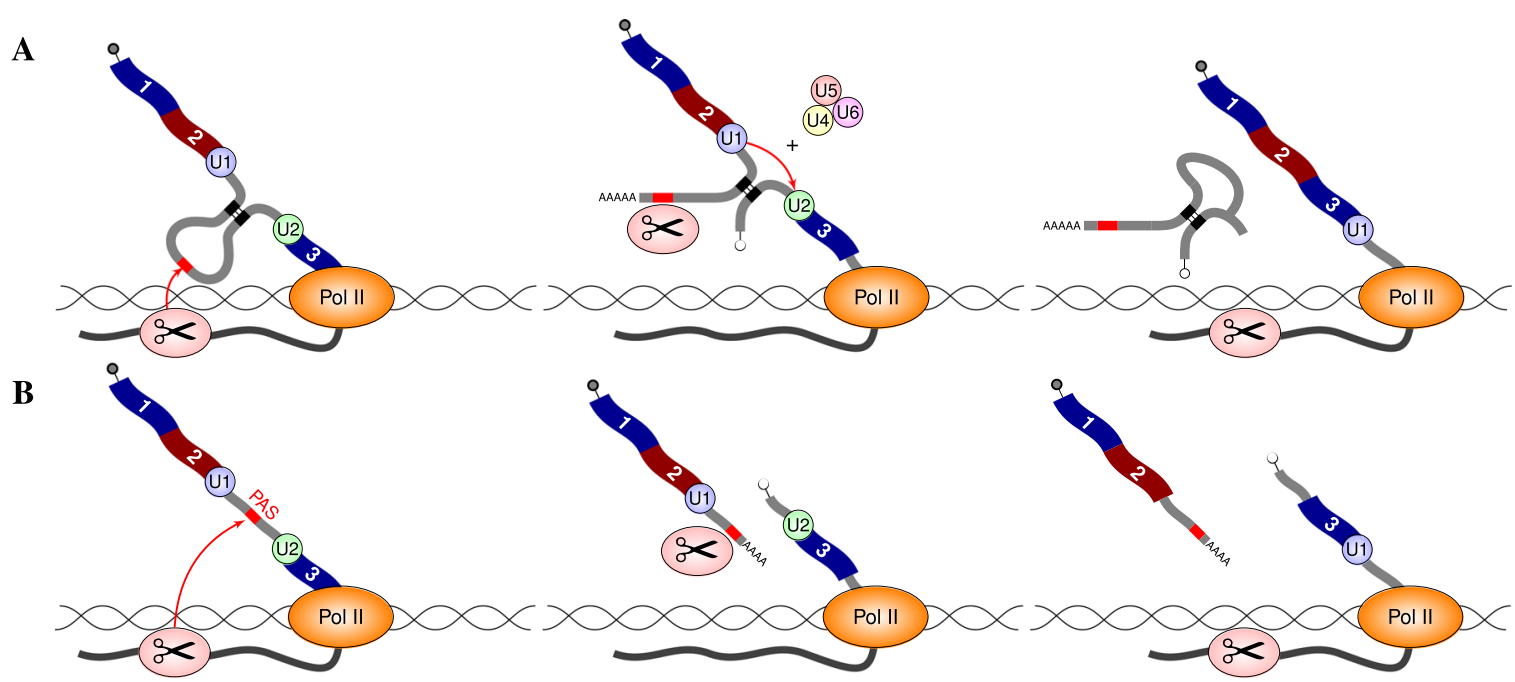

Fig. 7 RNA folding and splicing could mediate co-transcriptional suppression of premature cleavage and polyadenylation (a hypothesis). A The cleavage and polyadenylation of a structured pre-mRNA is rescued by the co-transcriptional splicing of the intron, while RNA structure stabilizes the molecule through intramolecular base pairings. B In the absence of RNA structure, such a rescue would not happen when splicing has a delay relative to cleavage and polyadenylation. Switching between $(\mathbf{A})$ and (B) depends on the rates of splicing, folding, and RNA Pol II elongation. 
strategies for profiling of RNA-RNA contacts such as RIC-seq ${ }^{24}$. This appears to be the most promising direction for future research.

In sum, eukaryotic pre-mRNAs are structured macromolecules with base pairings that span long distances, and RNA secondary structure plays a critical role in their processing. Here, we presented the most complete up-to-date catalog of CCRs in human protein-coding genes and provide their extensive characterization. In spite of the high false-positive rate, the predicted doublestranded RNA structures show significant associations with virtually all steps of pre-mRNA processing. We offer this catalog for common use as a reference set and provide its convenient visualization through a UCSC Genome Browser track hub.

\section{Methods \\ Genomes and transcript annotations. February 2009 (hg19, GRCh37) and} December 2013 (hg38, GRCh38) assemblies of the human genome were downloaded from Genome Reference Consortium ${ }^{97}$. These assemblies were used with GENCODE transcript annotations v19 and v33, respectively ${ }^{98}$. Only genes labeled "protein coding" were analyzed. Transcript annotations were parsed by custom scripts to extract the coordinates of exons and introns. The results for GRCh37 assembly are reported throughout the paper; however, Genome Browser track hubs (see below) contain tracks for both GRCh37 and GRCh38 assemblies.

Filtration of intronic regions. The intronic regions were defined as the longest continuous segments within genes that do not overlap any annotated exons (including exons of other genes). The intronic regions were extended by $10 \mathrm{nts}$ into the flanking exons to enable the identification of CCRs that overlap splice sites such as regions R1-R5 in the human Ate1 gene ${ }^{36}$. Next, these regions were intersected with the set of conserved RNA elements (phastConsElements track for the alignment of 99 vertebrates genomes to the human genome ${ }^{99}$ ) using bedtools ${ }^{100}$ We additionally excluded genomic regions that overlap intervals from the UCSC Genome Browser tRNA track ${ }^{101-105}$, sno/miRNA track ${ }^{106-112}$, TFBS Conserved track, which consists of Conserved Transcription Factor Binding Sites generated using the Transfac Matrix and Factor databases ${ }^{113}$, RepeatMasker track ${ }^{114}$ (all categories including SINE (short interspersed nuclear elements) and LINE (long interspersed nuclear elements)), SimpleTandemRepeats located by Tandem Repeats Finder ${ }^{115}$, and Human Nuclear mitochondrial sequences ${ }^{116-118}$. Genomic regions marked as "snRNA," "miRNA," "miscRNA," "snoRNA," "rRNA," and "tRNA" in GENCODE genome annotation were also excluded. The resulting set of CIRs was used as an input for the next step, which is described below.

PrePH. The first reference to long-range RNA structure appeared in the literature under the term "panhandle structure," which was coined in by virologists in the 1980 s to refer to a complementary base pairing between the $5^{\prime}$ end and $3^{\prime}$ end of the RNA genome of several segmented negative-stranded viruses ${ }^{119}$.

The PrePH utility uses a $k$-mer-based technique, which is similar to previously published IRBIS method ${ }^{29}$, to identify all pairs of nearly perfect complementary regions in a given pair of sequences. At the preparatory step, PrePH pre-computes a $4^{k} \times 4^{k}$ table containing helix hybridization energies for all pairs of $k$-mers (default $k=5$ ) that are either Watson-Crick complementary or contain a few GT base pairs (by default at most two) using energy tables from Vienna RNA package ${ }^{120}$. The dynamic programming matrix is computed by local Smith-Waterman algorithm using a limited set of structural rules: initiating a $k$-ntlong helix, extending a helix by a stacking base pair, and adding to a helix a short internal loop or bulge with up to $m$ nucleotides in each strand (default $m=2$ ), followed by another helix. To speed up back-tracking, PrePH uses auxiliary matrices to store intermediate structures, and reports nonintersecting pairs of complementary regions passing the energy threshold (by default $-15 \mathrm{kcal} / \mathrm{mol}$ ). Here, two pairs of complementary regions, $x_{1}$ complementary to $y_{1}$ and $x_{2}$ complementary to $y_{2}$, are referred to as intersecting if $x_{1}$ has common nucleotides with $x_{2}$ and also $y_{1}$ has common nucleotides with $y_{2}$. That is, two pairs of complementary regions may intersect by only one, not both interacting strands. The reduced scoring scheme, optimized back-tracking, and indexing of the initial sequences by $k$-mers result in a great improvement of computation speed. The detailed description of PrePH is exempt to Supplementary Methods. PrePH software is available at github (https://github.com/kalmSveta/PrePH) (codes are available at: https://doi.org/10.5281/zenodo.4601044).

Benchmark. We compared the accuracy and runtime of PrePH to those of other programs such as IntaRNA2.0 ${ }^{121}$, RIsearch2 ${ }^{122}$, RNAplex ${ }^{123}$, DuplexFold ${ }^{124}$, and bifold $^{124}$. PrePH was run with the following parameters: $k$-mer length is $5 \mathrm{nts}$, maximal distance between complementary regions is $10,000 \mathrm{nts}$, the minimal length of the aligned regions is $10 \mathrm{nts}$, the energy threshold is $-15 \mathrm{kcal} / \mathrm{mol}$, and the maximal number of Wobble pairs in a $k$-mer is 2 . The parameters for the other programs are listed below.
To benchmark the time efficiency, we use a set of 1000 pairs of randomly chosen conserved intronic sequences from the human genome. The sequences were 50 to $500 \mathrm{nts}$ long and contained nearly perfect sequence complementarity. All the programs were run with the energy threshold set to $-15 \mathrm{kcal} / \mathrm{mol}$. IntaRNA2.0 outOverlap parameter was set to $B$, which allowed overlap for interacting subsequences for both target and query; $n$ parameter was set to 100 to limit the maximal number of suboptimal structures; $q A c c$ and $t A c c$ were set to $\mathrm{N}$ to omit the computation of accessibility. RIsearch2 seed length was set to 5, the length of flanking sequences considered for seed extension was set to 50. RNAplex fastfolding parameter was set to $f 2$ to allow the structure to be computed based on the approximated model. DuplexFold and bifold maximum loop/bulge size was set to two. All other parameters were left at their default values. The computations were carried out on Intel R Core TM i5-8250U CPU with $1.60 \mathrm{GHz}$. PrePH showed the quickest result compared to the other programs (191.4 s) (Table S2A). At that, the equilibrium free energies of the predictions by PrePH correlated reasonably well with those of RNAplex, IntaRNA, and Duplexfold (Fig. S13).

For the comparison of MFE between different methods, we used simulated data with 1000 pairs of nearly perfect sequence complementarity, which were from 10 to $50 \mathrm{nts}$. All the programs were run with the energy threshold set to $-15 \mathrm{kcal} / \mathrm{mol}$. The other parameters were as before. Pearson's correlation coefficients were computed between energies of the predicted optimal structures. To compare the predictions of PrePH with predictions of other programs at the level of individual base pairs, we computed the following metric

$$
\text { Score }=\frac{\left|S_{1} \cap S_{2}\right|}{\left|S_{1}\right|}
$$

where $S_{1}$ is the set of base pairs predicted by $\mathrm{PrePH}, S_{2}$ is the set of base pairs predicted by the other program, and $S_{1} \cap S_{2}$ is the common set of base pairs $(|S|$ denotes the cardinality of a set). The number of base pairs that were common between PrePH and each of the other programs as a fraction of the number of base pairs predicted by PrePH alone was used as a measure of specificity (Table S2B). PrePH showed specificity $>80 \%$ with respect to all other programs except bifold; however, the latter was not in agreement with all other programs.

We conclude that PrePH allows for computationally efficient detection of PCCRs without significant loss of accuracy compared to other methods. The computation time of PrePH on the complete dataset of CIRs was $4 \mathrm{~h}$ (15 threads, $1200 \mathrm{MHz}$ CPUs each).

Relative position within the gene. The relative position of a genomic interval $[x$, $y]$ in the containing gene $[a, b]$, where $x, y, a$, and $b$ are genomic coordinates on the plus strand, was calculated as $p=\frac{x-a}{(y-x)-(b-a)+1}$ for the genes on the positive strand. For the genes on the negative strand the value of $1-p$ was used instead.

GO enrichment analysis. GO enrichment analysis was performed by controlling for the gene length since genes with PCCRs tend to be longer than genes without PCCRs. We randomly matched each gene with PCCRs to a gene without PCCR of approximately the same length. The enrichment of GO terms $s^{125,126}$ between these two gene sets was calculated with clusterProfiler R package ${ }^{127}$.

Experimental RNA structure data. The icSHAPE reactivity scores ${ }^{128}$ were mapped from GRCh38 to GRCh37 human genome assembly by LiftOver ${ }^{129}$. The reactivity score of a CCR was calculated as the average reactivity of its base-paired nucleotides, for which the icSHAPE reactivity score was available. The background reactivity was calculated as the average reactivity of the same number of nucleotides chosen at random outside the CCR, but in the same CIR. Wilcoxon's signedrank test was applied to matched samples of reactivity score differences to test for departures from zero.

The coordinates of base-paired regions from PARIS experiments ${ }^{22}(15,036$ pairs) were mapped from GRCh38 to GRCh37 by Liftover ${ }^{129}$. The coordinates of base-paired regions from LIGR-seq data (551,926 pairs) were used in the GRCh37 human genome assembly ${ }^{23}$. The coordinates of intramolecular base-paired regions from RIC-seq data ${ }^{24}(501,144$ pairs) were kindly provided by Prof. Xue by request (Supplementary Data File 5). In all three datasets, we selected the interacting pairs that were located intramolecularly within CIR of protein-coding genes from 1 to $10,000 \mathrm{nts}$ apart from each other. This resulted in 907 such pairs for PARIS, 586 for LIGR-seq, and 1804 for RIC-seq. In order to evaluate the precision and recall, we selected CIR with at least one nucleotide overlapping the experimentally validated structures and confined our analysis to PCCRs located in these regions. A CCR was classified as a true positive if it had at least one common nucleotide with an experimentally validated structure. A PCCR was classified as a true positive if both its CCRs intersected by at least one nucleotide with an experimentally validated such pair.

Cell culture, treatments, and RNA purification. A549 cell line (kindly gifted by Dr. Ilya Terenin, Moscow State University) was maintained in Dulbecco's modified Eagle's medium/Nutrient Mixture F-12 medium containing 10\% fetal bovine serum, $50 \mathrm{U} / \mathrm{ml}$ penicillin, and $0.05 \mathrm{mg} / \mathrm{ml}$ streptomycin (all products from Thermo Fisher Scientific) at $37^{\circ} \mathrm{C}$ in $5 \% \mathrm{CO}_{2}$. Cell line authentication was confirmed using short tandem repeat analysis. The cell line was tested for the absence 
of mycoplasma using MycoReport kit (EuroGene). For $\alpha$-amanitin (Sigma) treatments, 1 and $2 \mu \mathrm{g} / \mathrm{mL}$ of $\alpha$-amanitin was added to cells at $50-70 \%$ confluency. After $24 \mathrm{~h}$ of treatment, cells were harvested, total RNA was isolated using PureLink RNA Mini Kit (Thermo Fisher Scientific). Poly(A) ${ }^{+}$mRNA was purified using Dynabeads Oligo(dT) 25 (Thermo Fisher Scientific) following the manufacturer's instructions.

Library preparation and RNA-seq. Illumina cDNA libraries were constructed using NEBNext Ultra II Directional RNA Library Prep Kit for Illumina (New England BioLabs) following the manufacturer's protocol with the only modification: the change of fragmentation time from 15 to 10 min. Complementary DNA libraries were sequenced using the NextSeq500 (Illumina, San Diego, CA, USA) instrument; 33-41 million raw reads were obtained for each sample with a $75 \mathrm{bp}$ read length. The results of RNA-seq have been deposited at Gene Expression Omnibus under the accession number GSE153303.

Splicing quantification. RNA-seq data of poly(A) ${ }^{+}$RNA for the HepG2 cell line (accession numbers ENCFF670LIE and ENCFF074BOV) were downloaded in BAM format from the ENCODE Consortium website ${ }^{130}$. Short-hairpin shRNA$\mathrm{KD}$ of 250 RBPs followed by RNA-seq data ${ }^{65}$ were downloaded in BAM format from ENCODE data repository130,131 (Table S3). Poly(A)+ RNA from wild-type $A m r$ and Rpb1 C4/R749H mutant HEK293 cells treated with $\alpha$-amanitin for $42 \mathrm{~h}$ were downloaded from the Gene Expression Omnibus (GSE63375) ${ }^{71}$. RNA-seq data of poly(A) ${ }^{+}$RNA data from the A549 cell line treated with $\alpha$-amanitin were obtained as explained below and mapped to the GRCh37 human genome assembly using STAR aligner v2.3.1z with the default settings ${ }^{132}$. The coordinates of circRNAs expressed in liver tissue and their associated SRPTM metrics (the number of circular reads per number of mapped reads per read length) were obtained from the TCSD database ${ }^{47}$. The genomic coordinates of adenine branch point nucleotides were selected from the validated set of branch points expressed in K562 cells ${ }^{42}$.

RNA-seq experiments were processed by IPSA pipeline to obtain split read counts supporting splice junctions ${ }^{133}$. Split read counts were filtered by the entropy content of the offset distribution, annotation status, and canonical GT/AG dinucleotides at splice sites with the default settings, and pooled between bioreplicates. The exon inclusion rate ( $\Psi$, PSI, or Percent-Spliced-In) was calculated according to the equation

$$
\Psi=\frac{i n c}{i n c+2 \times e x c},
$$

where inc is the number of reads supporting exon inclusion and exc is the number of reads supporting exon exclusion. $\Psi$ values with the denominator $<10$ were considered unreliable and discarded. Differential exon inclusion between a pair of conditions (shRNA-KD vs. non-specific control and $\alpha$-amanitin vs. untreated control) was assessed as described previously ${ }^{134}$.

Cryptic and actively expressed splices sites in the human transcriptome were identified using genomic alignments of RNA-seq samples from the GTEx Consortium $^{43}$. Splice sites with the canonical GT/AG dinucleotides were called from split read alignments and ranked by the total number of supporting split reads pooled across all 8551 samples. The top $2 \%$ (respectively, bottom $2 \%$ ) of splice sites among those supported by at least three split reads were referred to as active (respectively, inactive). In order to identify cryptic splice sites, we applied the same strategy as in ref. ${ }^{135}$ by scanning the intron sequences for any sites that have a MaxEntScan score $>800$ for donor sites and $>950$ for acceptor sites ${ }^{136}$ and excluding splice sites that were detected in GTEx or present in the genome annotation. MaxEntScan score thresholds were chosen to have a comparable number of splice sites as in active and inactive sets above.

5'-end and 3'-end RNA processing. The genomic coordinates of human poly(A) sites profiled by high-throughput sequencing of $3^{\prime}$ ends of polyadenylated transcripts (poly(A)-seq) that were supported by 20 or more reads and intersected with the annotated transcript ends in GENCODE database were used ${ }^{57}$. Similarly, clusters of human CAGE tags expressed in the HepG2 cell line that were supported by RPKM of at least 10 and intersected with the annotated transcript starts in GENCODE database were used ${ }^{137}$.

RNA editing. A-to-I RNA-editing sites were obtained from RADAR ${ }^{53}$ and REDIportal ${ }^{54}$ databases. To compare the density of RNA editing sites in CCR and that in the adjacent conserved regions, we computed the number of adenosine residues that are RNA-editing sites within CCR and compared it to the respective figures for CIRs of the same length outside of CCR. OR was calculated for the contingency table of the number of adenosine residues that are/are not RNAediting sites within/outside CCR.

eCLIP. eCLIP peaks for $74 \mathrm{RBPs}$ assayed in HepG2 human cell line were downloaded from the ENCODE data repository in bed format ${ }^{65,130,131}$ (Table S4). The peaks were filtered by the conditions $\log \mathrm{FC} \geq 3$ and $p$ value $<0.001$. Since the agreement between two replicates was moderate, we use the pooled set of eCLIP peaks. To quantify the association between RBP binding and individual CCR, we calculated for each RBP the number of CCRs that intersected with its eCLIP peaks by at least $50 \%$ of the CCR length. This number was compared to the respective number of intersections obtained in the random shift control. To quantify the association of RBP binding with both CCRs in a PCCR, we constructed a contingency table for the number of PCCRs that had/did not have eCLIP peaks in left/ right CCR for each RBP, and computed the respective OR. The $95 \%$ confidence interval for the OR was calculated by Fisher's test.

Population polymorphisms. To evaluate the enrichment of population polymorphisms in CCR, we used genotyping data from phase 3 of the 1000 Genomes Project for 2504 individuals ${ }^{60}$ and computed the density of SNPs (single-nucleotide variants (SNVs) present in $>0.1 \%$ of individuals) in stacked nucleotide pairs of CCR and compared it to the density of SNPs in conserved regions outside CCRs (regions of each type were merged with bedtools merge ${ }^{138}$ before calculating the densities).

To assess the impact of SNPs on RNA structure stability, we calculated PCCR energy for the mutated sequence using energy parameters ${ }^{120}$ and compared it to the respective energy of the structure with SNPs of the same substitution type as observed originally, but introduced at a different position. We selected PCCRs with the free energy change $>2 \mathrm{kcal} / \mathrm{mol}$ by absolute value and compared the two energy sets using Wilcoxon's signed-rank test.

To evaluate the enrichment of compensatory mutations in PCCRs, we selected SNVs that occur in $>1 \%$ of donors in the 1000 Genome Project and intersected their list with the list of base pairs in PCCRs. Among them, we estimated the number of base pairs, in which a compensatory mutation occurred in $>1 \%$ of donors. To estimate the expected number of base pairs with compensatory mutations, we first subdivided base pairs into groups composed of the same base pair types (AT, TA, CG, GC, TG, GT) located on the same chromosome with the same number of SNP donors. Then, we randomly interchanged ("re-wired") base pairs within each group, for example, $A_{1} T_{1}$ and $A_{2} T_{2}$ were replaced by $A_{1} T_{2}$ and $\mathrm{A}_{2} \mathrm{~T}_{1}$ and applied the same procedure again, that is, estimated the number of base pairs, in which compensatory mutations defined by SNVs occurred in $>1 \%$ of donors (Fig. S6A). This randomization procedure was repeated 100 times.

Sequence conservation and complementary substitutions. To assess the degree of evolutionary conservation of a CCR, we computed the difference between the average PhastCons conservation score ${ }^{99}$ of all its nucleotides and the average PhastCons conservation score of the same number of nucleotides in its flanking regions within the same phastConsElements interval.

To assess the number of complementary substitutions in PCCRs and their statistical significance, we used global MSAs of 99 vertebrate genomes with human genome ${ }^{139}$. For each PCCR, we extracted two parts of the MSA corresponding to two CCRs using Bio.AlignIO.MafIO module from biopython library ${ }^{140}$. The organisms that had indels compared to the reference organism (hg19) in any of the two CCRs were removed. The number of orthologous sequences for each PCCR ranged from 15 to 99 . The two alignment blocks were merged through an additional spacer containing ten adenine nucleotides, resulting in an MSA STOCKHOLM format with a secondary RNA structure generated by PrePH. Next, we restrict the phylogenetic tree for the original MSA ${ }^{139}$ to have only the organisms available for the given PCCR and pass the tree and MSA to R-scape v1.2.3 ${ }^{40}$ with the following parameters: $-E 1-s-$ samplewc -nofigures. The output .out files of Rscape were parsed by custom scripts to extract $E$ values of individual base pairs. The $E$ value of the PCCR was defined to be equal to the product of $E$ values of the base pairs that were marked as having significant covariations by R-scape. As a result of this procedure, $E$ values were obtained for 909,146 PCCRs; 539,264 $E$ values for PCCRs that were $<1$ were adjusted using Benjamini-Hochberg correction. MSA and the phylogenetic trees were downloaded from the UCSC Genome Browser website (http://hgdownload.cse.ucsc.edu/goldenpath/hg19/ multiz100way/). Structural alignments were visualized using tableGrob function from gridExtra $\mathrm{R}$ package.

Statistical analysis. The data were analyzed and visualized using $\mathrm{R}$ statistics software version 3.4.1 and ggplot2 package. Nonparametric tests were performed by built-in $\mathrm{R}$ functions using normal approximation with continuity correction. MW denotes Mann-Whitney sum of ranks test. Boxplots in all figures are represented by the median, upper and lower quartiles, and upper and lower fences; outliers are not shown. Error bars in all figures and the numbers after the \pm sign represent $95 \%$ confidence intervals. Two-sided $p$ values are reported throughout the manuscript unless the contrary is specified. The levels of significance $5 \%, 1 \%$, and $0.1 \%$ in all figures are denoted by $*, * *$, and $* * *$, respectively.

Reporting summary. Further information on research design is available in the Nature Research Reporting Summary linked to this article.

\section{Data availability}

The RNA-seq data generated in this study are available through GEO repository under the accession code GSE153303. Public data that were analyzed in this study are available via accession codes listed in "Methods" and Supplementary information. Supplementary 
Data Files are available online through a repository at https://zenodo.org/record/4603132 (also see https://doi.org/10.5281/zenodo.4603132). All relevant intermediate data files are available from the authors upon request.

\section{Code availability}

The software developed in this study is available at https://github.com/kalmSveta/PrePH (codes are available at: https://doi.org/10.5281/zenodo.4601044).

Received: 13 May 2020; Accepted: 20 March 2021;

Published online: 16 April 2021

\section{References}

1. Breaker, R. R. Riboswitches and the RNA world. Cold Spring Harb. Perspect. Biol. 4, a003566 (2012).

2. Bowman, J. C., Hud, N. V. \& Williams, L. D. The ribosome challenge to the RNA world. J. Mol. Evol. 80, 143-161 (2015).

3. Quinn, J. J. \& Chang, H. Y. Unique features of long non-coding RNA biogenesis and function. Nat. Rev. Genet. 17, 47-62 (2016).

4. Marchese, F. P., Raimondi, I. \& Huarte, M. The multidimensional mechanisms of long noncoding RNA function. Genome Biol. 18, 206 (2017).

5. Guttman, M. \& Rinn, J. L. Modular regulatory principles of large non-coding RNAs. Nature 482, 339-346 (2012).

6. Silverman, I. M., Li, F. \& Gregory, B. D. Genomic era analyses of RNA secondary structure and RNA-binding proteins reveal their significance to post-transcriptional regulation in plants. Plant Sci. 205-206, 55-62 (2013).

7. Baralle, F. E., Singh, R. N. \& Stamm, S. RNA structure and splicing regulation. Biochim. Biophys. Acta Gene Regul. Mech. 1862, 194448 (2019).

8. Wu, X. \& Bartel, D. P. Widespread influence of 3'-end structures on mammalian mRNA processing and stability. Cell 169, 905-917 (2017).

9. Rieder, L. E. \& Reenan, R. A. The intricate relationship between RNA structure, editing, and splicing. Semin. Cell Dev. Biol. 23, 281-288 (2012).

10. Garcia-Lopez, A. et al. Targeting RNA structure in SMN2 reverses spinal muscular atrophy molecular phenotypes. Nat. Commun. 9, 2032 (2018).

11. Leppek, K., Das, R. \& Barna, M. Functional 5' UTR mRNA structures in eukaryotic translation regulation and how to find them. Nat. Rev. Mol. Cell Biol. 19, 158-174 (2018).

12. Bernat, V. \& Disney, M. D. RNA structures as mediators of neurological diseases and as drug targets. Neuron 87, 28-46 (2015).

13. Singh, N. N. \& Singh, R. N. How RNA structure dictates the usage of a critical exon of spinal muscular atrophy gene. Biochim. Biophys. Acta Gene Regul. Mech. 1862, 194403 (2019).

14. Pervouchine, D. D. Towards long-range RNA structure prediction in eukaryotic genes. Genes 9, 302 (2018).

15. Ding, Y. et al. In vivo genome-wide profiling of RNA secondary structure reveals novel regulatory features. Nature 505, 696-700 (2014).

16. Rouskin, S., Zubradt, M., Washietl, S., Kellis, M. \& Weissman, J. S. Genomewide probing of RNA structure reveals active unfolding of mRNA structures in vivo. Nature 505, 701-705 (2014).

17. Spitale, R. C. et al. Structural imprints in vivo decode RNA regulatory mechanisms. Nature 519, 486-490 (2015).

18. Flynn, R. A. et al. Transcriptome-wide interrogation of RNA secondary structure in living cells with icSHAPE. Nat. Protoc. 11, 273-290 (2016).

19. Wan, Y. et al. Landscape and variation of RNA secondary structure across the human transcriptome. Nature 505, 706-709 (2014)

20. Ramani, V., Qiu, R. \& Shendure, J. High-throughput determination of RNA structure by proximity ligation. Nat. Biotechnol. 33, 980-984 (2015).

21. Aw, J. G. et al. In vivo mapping of eukaryotic RNA interactomes reveals principles of higher-order organization and regulation. Mol. Cell 62, 603-617 (2016).

22. Lu, Z. et al. RNA duplex map in living cells reveals higher-order transcriptome structure. Cell 165, 1267-1279 (2016).

23. Sharma, E., Sterne-Weiler, T., O'Hanlon, D. \& Blencowe, B. J. Global mapping of human RNA-RNA interactions. Mol. Cell 62, 618-626 (2016).

24. Cai, Z. et al. RIC-seq for global in situ profiling of RNA-RNA spatial interactions. Nature 582, 432-437 (2020).

25. Pedersen, J. S. et al. Identification and classification of conserved RNA secondary structures in the human genome. PLoS Comput. Biol. 2, e33 (2006).

26. Rivas, E., Clements, J. \& Eddy, S. R. Estimating the power of sequence covariation for detecting conserved RNA structure. Bioinformatics 36, 3072-3076 (2020).

27. Raker, V. A., Mironov, A. A., Gelfand, M. S. \& Pervouchine, D. D. Modulation of alternative splicing by long-range RNA structures in Drosophila. Nucleic Acids Res. 37, 4533-4544 (2009).
28. Pervouchine, D. D. et al. Evidence for widespread association of mammalian splicing and conserved long-range RNA structures. RNA 18, 1-15 (2012).

29. Pervouchine, D. D. IRBIS: a systematic search for conserved complementarity. RNA 20, 1519-1531 (2014)

30. Will, S., Yu, M. \& Berger, B. Structure-based whole-genome realignment reveals many novel noncoding RNAs. Genome Res. 23, 1018-1027 (2013).

31. Raney, B. J. et al. Track data hubs enable visualization of user-defined genome-wide annotations on the UCSC Genome Browser. Bioinformatics 30, 1003-1005 (2014).

32. Lovci, M. T. et al. Rbfox proteins regulate alternative mRNA splicing through evolutionarily conserved RNA bridges. Nat. Struct. Mol. Biol. 20, 1434-1442 (2013).

33. Solnick, D. \& Lee, S. I. Amount of RNA secondary structure required to induce an alternative splice. Mol. Cell. Biol. 7, 3194-3198 (1987).

34. Margulies, E. H., Blanchette, M., Haussler, D. \& Green, E. D. Identification and characterization of multi-species conserved sequences. Genome Res. 13, 2507-2518 (2003)

35. Taube, J. R. et al. PMD patient mutations reveal a long-distance intronic interaction that regulates PLP1/DM20 alternative splicing. Hum. Mol. Genet. 23, 5464-5478 (2014)

36. Kalinina, M. et al. Multiple competing RNA structures dynamically control alternative splicing in the human ATE1 gene. Nucleic Acids Res. 49, 479-490 (2021).

37. Kirby, D. A., Muse, S. V. \& Stephan, W. Maintenance of pre-mRNA secondary structure by epistatic selection. Proc. Natl Acad. Sci. USA 92, 9047-9051 (1995).

38. Wilke, C. O., Lenski, R. E. \& Adami, C. Compensatory mutations cause excess of antagonistic epistasis in RNA secondary structure folding. BMC Evol. Biol. 3, 3 (2003).

39. Kern, A. D. \& Kondrashov, F. A. Mechanisms and convergence of compensatory evolution in mammalian mitochondrial tRNAs. Nat. Genet. 36 1207-1212 (2004).

40. Rivas, E., Clements, J. \& Eddy, S. R. A statistical test for conserved RNA structure shows lack of evidence for structure in IncRNAs. Nat. Methods 14, 45-48 (2017).

41. Li, P., Shi, R. \& Zhang, Q. C. icSHAPE-pipe: a comprehensive toolkit for icSHAPE data analysis and evaluation. Methods 178, 96-103 (2020).

42. Mercer, T. R. et al. Genome-wide discovery of human splicing branchpoints. Genome Res. 25, 290-303 (2015).

43. Melé, M. et al. Human genomics. The human transcriptome across tissues and individuals. Science 348, 660-665 (2015).

44. Deshler, J. O. \& Rossi, J. J. Unexpected point mutations activate cryptic 3' splice sites by perturbing a natural secondary structure within a yeast intron. Genes Dev. 5, 1252-1263 (1991)

45. Buratti, E. \& Baralle, F. E. Influence of RNA secondary structure on the premRNA splicing process. Mol. Cell. Biol. 24, 10505-10514 (2004).

46. Ottesen, E. W., Luo, D., Seo, J., Singh, N. N. \& Singh, R. N. Human survival motor neuron genes generate a vast repertoire of circular RNAs. Nucleic Acids Res. 47, 2884-2905 (2019).

47. Xia, S. et al. Comprehensive characterization of tissue-specific circular RNAs in the human and mouse genomes. Brief. Bioinform. 18, 984-992 (2017).

48. Walkley, C. R. \& Li, J. B. Rewriting the transcriptome: adenosine-to-inosine RNA editing by ADARs. Genome Biol. 18, 205 (2017).

49. Bass, B. L. RNA editing by adenosine deaminases that act on RNA. Annu. Rev. Biochem. 71, 817-846 (2002).

50. Hogg, M., Paro, S., Keegan, L. P. \& O'Connell, M. A. RNA editing by mammalian ADARs. Adv. Genet. 73, 87-120 (2011).

51. Nishikura, K. Functions and regulation of RNA editing by ADAR deaminases. Annu. Rev. Biochem. 79, 321-349 (2010).

52. Liddicoat, B. J. et al. RNA editing by ADAR1 prevents MDA5 sensing of endogenous dsRNA as nonself. Science 349, 1115-1120 (2015).

53. Ramaswami, G. \& Li, J. B. RADAR: a rigorously annotated database of A-to-I RNA editing. Nucleic Acids Res. 42, D109-113 (2014).

54. Picardi, E., D’Erchia, A. M., Lo Giudice, C. \& Pesole, G. REDIportal: a comprehensive database of A-to-I RNA editing events in humans. Nucleic Acids Res. 45, D750-D757 (2017)

55. Pan, H. et al. Competing RNA pairings in complex alternative splicing of a 3 variable region. RNA 24, 1466-1480 (2018).

56. Wright, J. C. et al. Improving GENCODE reference gene annotation using a high-stringency proteogenomics workflow. Nat. Commun. 7, 11778 (2016).

57. Derti, A. et al. A quantitative atlas of polyadenylation in five mammals. Genome Res. 22, 1173-1183 (2012).

58. Fejes-Toth, K. et al. Post-transcriptional processing generates a diversity of 5'modified long and short RNAs. Nature 457, 1028-1032 (2009).

59. Chamary, J. V. \& Hurst, L. D. Evidence for selection on synonymous mutations affecting stability of mRNA secondary structure in mammals. Genome Biol. 6, R75 (2005). 
60. Auton, A. et al. A global reference for human genetic variation. Nature 526, 68-74 (2015)

61. Taliaferro, J. M. et al. RNA sequence context effects measured in vitro predict in vivo protein binding and regulation. Mol. Cell 64, 294-306 (2016).

62. Kazan, H., Ray, D., Chan, E. T., Hughes, T. R. \& Morris, Q. RNAcontext: a new method for learning the sequence and structure binding preferences of RNA-binding proteins. PLoS Comput. Biol. 6, e1000832 (2010).

63. Li, X., Quon, G., Lipshitz, H. D. \& Morris, Q. Predicting in vivo binding sites of RNA-binding proteins using mRNA secondary structure. RNA 16, 1096-1107 (2010).

64. Van Nostrand, E. L. et al. Robust transcriptome-wide discovery of RNAbinding protein binding sites with enhanced CLIP (eCLIP). Nat. Methods 13, 508-514 (2016).

65. Van Nostrand, E. L. et al. A large-scale binding and functional map of human RNA-binding proteins. Nature 583, 711-719 (2020).

66. Dominguez, D. et al. Sequence, structure, and context preferences of human RNA binding proteins. Mol. Cell 70, 854-867 (2018).

67. Huang, $\mathrm{H}$. et al. Tissue-selective restriction of RNA editing of CaV1.3 by splicing factor SRSF9. Nucleic Acids Res. 46, 7323-7338 (2018).

68. Gaudet, P., Livstone, M. S., Lewis, S. E. \& Thomas, P. D. Phylogenetic-based propagation of functional annotations within the Gene Ontology consortium. Brief. Bioinform. 12, 449-462 (2011).

69. Ding, J. et al. Crystal structure of the two-RRM domain of hnRNP A1 (UP1) complexed with single-stranded telomeric DNA. Genes Dev. 13, 1102-1115 (1999).

70. Wang, X., Schwartz, J. C. \& Cech, T. R. Nucleic acid-binding specificity of human FUS protein. Nucleic Acids Res. 43, 7535-7543 (2015).

71. Fong, N. et al. Pre-mRNA splicing is facilitated by an optimal RNA polymerase II elongation rate. Genes Dev. 28, 2663-2676 (2014).

72. Schor, I. E., Gómez Acuña, L. I. \& Kornblihtt, A. R. Coupling between transcription and alternative splicing. Cancer Treat. Res. 158, 1-24 (2013).

73. Saldi, T., Cortazar, M. A., Sheridan, R. M. \& Bentley, D. L. Coupling of RNA polymerase II transcription elongation with pre-mRNA splicing. J. Mol. Biol. 428, 2623-2635 (2016).

74. Rudd, M. D. \& Luse, D. S. Amanitin greatly reduces the rate of transcription by RNA polymerase II ternary complexes but fails to inhibit some transcript cleavage modes. J. Biol. Chem. 271, 21549-21558 (1996).

75. Shirakawa, R. et al. Tuberous sclerosis tumor suppressor complex-like complexes act as GTPase-activating proteins for Ral GTPases. J. Biol. Chem. 284, 21580-21588 (2009).

76. Pace, C. N. Conformational stability of globular proteins. Trends Biochem. Sci. 15, 14-17 (1990)

77. Warf, M. B. \& Berglund, J. A. Role of RNA structure in regulating pre-mRNA splicing. Trends Biochem. Sci. 35, 169-178 (2010).

78. Welden, J. R. \& Stamm, S. Pre-mRNA structures forming circular RNAs. Biochim. Biophys. Acta Gene Regul. Mech. 1862, 194410 (2019).

79. Goguel, V., Wang, Y. \& Rosbash, M. Short artificial hairpins sequester splicing signals and inhibit yeast pre-mRNA splicing. Mol. Cell. Biol. 13, 6841-6848 (1993).

80. Jacquenet, S. et al. Conserved stem-loop structures in the HIV-1 RNA region containing the A3 3' splice site and its cis-regulatory element: possible involvement in RNA splicing. Nucleic Acids Res. 29, 464-478 (2001).

81. Estes, P. A., Cooke, N. E. \& Liebhaber, S. A. A native RNA secondary structure controls alternative splice-site selection and generates two human growth hormone isoforms. J. Biol. Chem. 267, 14902-14908 (1992).

82. Singh, N. N., Singh, R. N. \& Androphy, E. J. Modulating role of RNA structure in alternative splicing of a critical exon in the spinal muscular atrophy genes. Nucleic Acids Res. 35, 371-389 (2007).

83. McManus, C. J. \& Graveley, B. R. RNA structure and the mechanisms of alternative splicing. Curr. Opin. Genet. Dev. 21, 373-379 (2011).

84. Ramaswami, G. et al. Genetic mapping uncovers cis-regulatory landscape of RNA editing. Nat. Commun. 6, 8194 (2015).

85. de la Mata, M. et al. RNA polymerase II elongation at the crossroads of transcription and alternative splicing. Genet Res. Int. 2011, 309865 (2011).

86. de la Mata, M. et al. A slow RNA polymerase II affects alternative splicing in vivo. Mol. Cell 12, 525-532 (2003).

87. Dujardin, G. et al. How slow RNA polymerase II elongation favors alternative exon skipping. Mol. Cell 54, 683-690 (2014).

88. Pinto, P. A. et al. RNA polymerase II kinetics in polo polyadenylation signal selection. EMBO J. 30, 2431-2444 (2011)

89. Wright, S. Regulation of eukaryotic gene expression by transcriptional attenuation. Mol. Biol. Cell 4, 661-668 (1993).

90. Naville, M. \& Gautheret, D. Transcription attenuation in bacteria: theme and variations. Brief. Funct. Genom. Proteomic 8, 482-492 (2009).

91. Graveley, B. R., Fleming, E. S. \& Gilmartin, G. M. RNA structure is a critical determinant of poly(A) site recognition by cleavage and polyadenylation specificity factor. Mol. Cell. Biol. 16, 4942-4951 (1996).
92. Bentley, D. L. Coupling mRNA processing with transcription in time and space. Nat. Rev. Genet. 15, 163-175 (2014).

93. Movassat, M. et al. Coupling between alternative polyadenylation and alternative splicing is limited to terminal introns. RNA Biol. 13, 646-655 (2016).

94. Oh, J. M. et al. U1 snRNP telescripting regulates a size-function-stratified human genome. Nat. Struct. Mol. Biol. 24, 993-999 (2017).

95. Yao, C. et al. Transcriptome-wide analyses of CstF64-RNA interactions in global regulation of mRNA alternative polyadenylation. Proc. Natl Acad. Sci. USA 109, 18773-18778 (2012).

96. Van Nostrand, E. L., Huelga, S. C. \& Yeo, G. W. Experimental and computational considerations in the study of RNA-binding protein-RNA interactions. Adv. Exp. Med. Biol. 907, 1-28 (2016).

97. Church, D. M. et al. Modernizing reference genome assemblies. PLoS Biol. 9, e1001091 (2011).

98. Harrow, J. et al. GENCODE: the reference human genome annotation for The ENCODE Project. Genome Res. 22, 1760-1774 (2012).

99. Siepel, A. et al. Evolutionarily conserved elements in vertebrate, insect, worm, and yeast genomes. Genome Res. 15, 1034-1050 (2005).

100. Quinlan, A. R. \& Hall, I. M. BEDTools: a flexible suite of utilities for comparing genomic features. Bioinformatics 26, 841-842 (2010).

101. Chan, P. P. \& Lowe, T. M. GtRNAdb: a database of transfer RNA genes detected in genomic sequence. Nucleic Acids Res. 37, D93-97 (2009).

102. Eddy, S. R. \& Durbin, R. RNA sequence analysis using covariance models. Nucleic Acids Res. 22, 2079-2088 (1994).

103. Fichant, G. A. \& Burks, C. Identifying potential tRNA genes in genomic DNA sequences. J. Mol. Biol. 220, 659-671 (1991).

104. Lowe, T. M. \& Eddy, S. R. tRNAscan-SE: a program for improved detection of transfer RNA genes in genomic sequence. Nucleic Acids Res. 25, 955-964 (1997).

105. Pavesi, A., Conterio, F., Bolchi, A., Dieci, G. \& Ottonello, S. Identification of new eukaryotic tRNA genes in genomic DNA databases by a multistep weight matrix analysis of transcriptional control regions. Nucleic Acids Res. 22, 1247-1256 (1994).

106. Griffiths-Jones, S. The microRNA Registry. Nucleic Acids Res. 32, D109-111 (2004).

107. Griffiths-Jones, S., Grocock, R. J., van Dongen, S., Bateman, A. \& Enright, A. J. miRBase: microRNA sequences, targets and gene nomenclature. Nucleic Acids Res. 34, D140-144 (2006).

108. Griffiths-Jones, S., Saini, H. K., van Dongen, S. \& Enright, A. J. miRBase: tools for microRNA genomics. Nucleic Acids Res. 36, D154-158 (2008).

109. Lestrade, L. \& Weber, M. J. snoRNA-LBME-db, a comprehensive database of human H/ACA and C/D box snoRNAs. Nucleic Acids Res. 34, D158-162 (2006).

110. Weber, M. J. New human and mouse microRNA genes found by homology search. FEBS J. 272, 59-73 (2005).

111. Kent, W. J. BLAT-the BLAST-like alignment tool. Genome Res. 12, 656-664 (2002).

112. Ambros, V. et al. A uniform system for microRNA annotation. RNA 9, 277-279 (2003).

113. Kent, W. J. et al. The human genome browser at UCSC. Genome Res. 12, 996-1006 (2002).

114. Jurka, J. Repbase update: a database and an electronic journal of repetitive elements. Trends Genet. 16, 418-420 (2000).

115. Benson, G. Tandem repeats finder: a program to analyze DNA sequences. Nucleic Acids Res. 27, 573-580 (1999).

116. Kidd, J. M. et al. Mapping and sequencing of structural variation from eight human genomes. Nature 453, 56-64 (2008).

117. Lascaro, D. et al. The RHNumtS compilation: features and bioinformatics approaches to locate and quantify Human NumtS. BMC Genomics 9, 267 (2008).

118. Simone, D., Calabrese, F. M., Lang, M., Gasparre, G. \& Attimonelli, M. The reference human nuclear mitochondrial sequences compilation validated and implemented on the UCSC genome browser. BMC Genomics 12, 517 (2011).

119. Hsu, M. T., Parvin, J. D., Gupta, S., Krystal, M. \& Palese, P. Genomic RNAs of influenza viruses are held in a circular conformation in virions and in infected cells by a terminal panhandle. Proc. Natl Acad. Sci. USA 84, 8140-8144 (1987).

120. Lorenz, R. et al. ViennaRNA Package 2.0. Algorithms Mol. Biol. 6, 26 (2011)

121. Mann, M., Wright, P. R. \& Backofen, R. IntaRNA 2.0: enhanced and customizable prediction of RNA-RNA interactions. Nucleic Acids Res. 45, W435-W439 (2017).

122. Alkan, F. et al. RIsearch2: suffix array-based large-scale prediction of RNARNA interactions and siRNA off-targets. Nucleic Acids Res. 45, e60 (2017)

123. Tafer, H. \& Hofacker, I. L. RNAplex: a fast tool for RNA-RNA interaction search. Bioinformatics 24, 2657-2663 (2008).

124. Reuter, J. S. \& Mathews, D. H. RNAstructure: software for RNA secondary structure prediction and analysis. BMC Bioinform. 11, 129 (2010) 
125. Ashburner, M. et al. Gene ontology: tool for the unification of biology. The Gene Ontology Consortium. Nat. Genet. 25, 25-29 (2000).

126. authors listed, N. The Gene Ontology Resource: 20 years and still GOing strong. Nucleic Acids Res. 47, D330-D338 (2019).

127. Yu, G., Wang, L. G., Han, Y. \& He, Q. Y. clusterProfiler: an R package for comparing biological themes among gene clusters. OMICS 16, 284-287 (2012).

128. Sun, L. et al. RNA structure maps across mammalian cellular compartments. Nat. Struct. Mol. Biol. 26, 322-330 (2019).

129. Hinrichs, A. S. et al. The UCSC Genome Browser Database: update 2006. Nucleic Acids Res. 34, D590-598 (2006).

130. Sloan, C. A. et al. ENCODE data at the ENCODE portal. Nucleic Acids Res. 44, D726-732 (2016).

131. Dunham, I. et al. An integrated encyclopedia of DNA elements in the human genome. Nature 489, 57-74 (2012).

132. Dobin, A. et al. STAR: ultrafast universal RNA-seq aligner. Bioinformatics 29, 15-21 (2013)

133. Pervouchine, D. D., Knowles, D. G. \& Guigó, R. Intron-centric estimation of alternative splicing from RNA-seq data. Bioinformatics 29, 273-274 (2013).

134. Pervouchine, D. et al. Integrative transcriptomic analysis suggests new autoregulatory splicing events coupled with nonsense-mediated mRNA decay. Nucleic Acids Res. 47, 5293-5306 (2019).

135. Bretschneider, H., Gandhi, S., Deshwar, A. G., Zuberi, K. \& Frey, B. J. COSSMO: predicting competitive alternative splice site selection using deep learning. Bioinformatics 34, i429-i437 (2018).

136. Yeo, G. \& Burge, C. B. Maximum entropy modeling of short sequence motifs with applications to RNA splicing signals. J. Comput. Biol. 11, 377-394 (2004).

137. Djebali, S. et al. Landscape of transcription in human cells. Nature 489 101-108 (2012).

138. Quinlan, A. R. BEDTools: the Swiss-Army Tool for genome feature analysis. Curr. Protoc. Bioinform. 47, 1-34 (2014).

139. Blanchette, M. et al. Aligning multiple genomic sequences with the threaded blockset aligner. Genome Res. 14, 708-715 (2004).

140. Cock, P. J. et al. Biopython: freely available Python tools for computational molecular biology and bioinformatics. Bioinformatics 25, 1422-1423 (2009).

141. Suyama, M. Mechanistic insights into mutually exclusive splicing in dynamin 1. Bioinformatics 29, 2084-2087 (2013)

\section{Acknowledgements}

RNA-sequencing was funded by Skolkovo Institute of Science and Technology. We thank Skoltech Genomics Core facility staff (Margarita Ezhova, Maria Logacheva) for library preparation and sequencing, and the Cobrain computer cluster for access to computational facilities. We also thank Prof. Yuanchao Xue and Prof. Changchang Cao for providing the list of clusters of chimeric reads for intramolecular interactions. S.K., S.D., and D.P. were supported by Russian Foundation for Basic Research Grant 18-29-13020 MK and by Skolkovo Institute of Science Technology Research Grant RF-0000000653. M.K. and D.S. were supported by Russian Foundation for Basic Research Grant 18-29 13020-MK.

\section{Author contributions}

D.P. and S.K. designed and carried out the analysis. M.K. and D.S. performed the experiments. S.D. and A.M. analyzed the evolutionary part. S.K., M.K., S.D., A.M., D.S., R.G., and D.P. analyzed the data and discussed the results. D.P. and S.K. wrote the paper.

\section{Competing interests}

The authors declare no competing interests.

\section{Additional information}

Supplementary information The online version contains supplementary material available at https://doi.org/10.1038/s41467-021-22549-7.

Correspondence and requests for materials should be addressed to D.P.

Peer review information Nature Communications thanks Daniel Gautheret and Yongfeng Jin for their contributions to the peer review of this work. Peer review reports are available.

Reprints and permission information is available at http://www.nature.com/reprints

Publisher's note Springer Nature remains neutral with regard to jurisdictional claims in published maps and institutional affiliations.

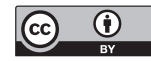

Open Access This article is licensed under a Creative Commons Attribution 4.0 International License, which permits use, sharing, adaptation, distribution and reproduction in any medium or format, as long as you give appropriate credit to the original author(s) and the source, provide a link to the Creative Commons license, and indicate if changes were made. The images or other third party material in this article are included in the article's Creative Commons license, unless indicated otherwise in a credit line to the material. If material is not included in the article's Creative Commons license and your intended use is not permitted by statutory regulation or exceeds the permitted use, you will need to obtain permission directly from the copyright holder. To view a copy of this license, visit http://creativecommons.org/ licenses/by/4.0/.

(C) The Author(s) 2021 